August, 2002

hep-th/0208139

\title{
Harmonic Forms and Deformation of ALC metrics with $\operatorname{Spin}(7)$ holonomy
}

\author{
Hiroaki Kanno⿵ \\ Graduate School of Mathematics \\ Nagoya University, Nagoya, 464-8602, Japan \\ Yukinori Yasui \\ Department of Physics, Osaka City University \\ Sumiyoshi-ku, Osaka, 558-8585, Japan
}

\begin{abstract}
Asymptotically locally conical (ALC) metric of exceptional holonomy has an asymptotic circle bundle structure that accommodates the $M$ theory circle in type IIA reduction. Taking $\operatorname{Spin}(7)$ metrics of cohomogeneity one as explicit examples, we investigate deformations of ALC metrics, in particular that change the asymptotic $S^{1}$ radius related to the type IIA string coupling constant. When the canonical four form of $\operatorname{Spin}(7)$ holonomy is taken to be anti-self-dual, the deformations of $\operatorname{Spin}(7)$ metric are related to the harmonic self-dual four forms, which are given by solutions to a system of first order differential equations, due to the metric ansatz of cohomogeneity one. We identify the $L^{2}$-normalizable solution that deforms the asymptotic radius of the $M$ theory circle.
\end{abstract}

*e-mail: kanno@math.nagoya-u.ac.jp

†e-mail: yasui@sci.osaka-cu.ac.jp 


\section{Introduction}

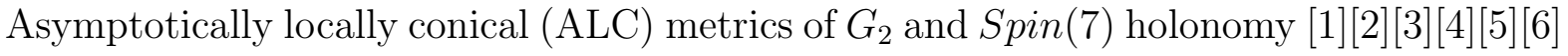
are expected to play an important role in $M$ theory compactification with minimal supersymmetry. The ALC metric has a circle bundle structure and the radius of the $S^{1}$ fiber stabilizes asymptotically. Hence ALC metrics with exceptional holonomy may be regarded as higher dimensional generalizations of ALF metrics in four dimensions, typical examples of which are the Taub-NUT metric and the Atiyah-Hitchin metric. In $M$ theory compactification the asymptotic circle of ALC metric is identified with $M$ theory circle whose radius is related to the string coupling of type IIA reduction. In general ALC metric has a modulus of asymptotic radius of the $S^{1}$ fiber other than the modulus of overall scaling of Ricci-flat metric. By deforming the radius of the asymptotic circle with the overall scale parameter being fixed, we can interpolate the strong coupling $M$ theory limit and the weak coupling region of perturbative IIA string theory. Namely when the radius goes to infinity, an asymptotic conical (AC) metric arises as a limit of ALC metric and it describes a purely gravitational background of $M$ theory. The limit of the other side is what is called the Gromov-Hausdorff limit and the limiting metric is a direct product of a Ricci-flat metric of lower dimensions and the Euclidean metric. Such a picture gives a beautiful $M$ theory unification [7] of the resolved conifold and the deformed conifold of IIA background that has been employed to uncover the strong coupling dynamics of $N=1$ supersymmetric gauge theory in four dimensions [8] [10]. (See also [11] for an early attempt.) The AC metric in the strong coupling limit is a resolution of the cone metric over $S U(2)^{3} / S U(2)$ that possesses a triality symmetry 10. Each of three metrics permutated by the triality has a different Gromov-Haussdorff limit whose Calabi-Yau part is identified as either the deformed conifold or one of the two resolved conifold metrics. They are different resolutions of the cone metric over $S U(2)^{2} / U(1)$ related by flop operation.

In this article we will investigate the deformation of ALC metrics of Spin(7) holonomy with a view to $M$ theory compactification described above. It is known that the metric of $\operatorname{Spin}(7)$ holonomy is characterized by a closed four form

$$
\Omega=\Omega_{a b c d} e^{a} \wedge e^{b} \wedge e^{c} \wedge e^{d}
$$

where $\left\{e^{a}\right\}$ is a vielbein (an orthonormal frame) and $\Omega_{a b c d}$ is related to the structure 
constants of octonions [12]. We will take a convention that the canonical four form $\Omega$ is anti-self-dual. Then the formal dimensions of the moduli space of metric of $\operatorname{Spin}(7)$ holonomy is $1+b_{4}^{+}$, where $b_{4}^{+}$is the Betti number of self-dual four form [13] [12]. Note that the overall scaling of Ricci-flat metric always gives one dimensional modulus. The appearance of $b_{4}^{+}$is understood as follows; In eight dimensions the representation of the traceless part of metric tensor is $\mathbf{3} \mathbf{5}_{V}$ of $S O(8)$. When we have a metric of $\operatorname{Spin}(7)$ holonomy, there is a covariantly constant spinor in $\mathbf{8}_{C}$ of $S O(8)$ and we have a global identification of the vector representation and the spinor representation $\mathbf{8}_{V} \equiv \mathbf{8}_{S}$. This implies a further identification of $35_{V}$ with the representation $35_{S}$ of the self-dual four form. On the other hand the representation $35_{C}$ of the anti-self-dual four form is reducible and decomposed as $\mathbf{1} \oplus \mathbf{7} \oplus \mathbf{2 7}$, because the conjugate spinor bundle is reducible ( $\mathbf{8}_{C}=$ $1 \oplus \mathbf{7})$ on the manifold of $\operatorname{Spin}(7)$ holonomy. The seven dimensional invariant subspace in $35_{C}$ is specified by the canonical anti-self-dual four form $\Omega$ defined by (1.1). When a self-dual four form in $\mathbf{3 5} \mathbf{5}_{S}$ is closed and hence harmonic, the corresponding infinitesimal deformation of the traceless part of metric in $\mathbf{3} \mathbf{5}_{V}$ does not break the Ricci-flatness. Therefore, we can define infinitesimal deformations of the traceless part of Spin(7) metric from harmonic self-dual four forms. Precisely speaking this argument is valid for compact manifolds. In this paper we consider Spin(7) metrics of cohomogeneity one and they are metrics on non-compact manifolds. Our claim of the correspondence of harmonic selfdual four forms and deformations of $\operatorname{Spin}(7)$ metric remains formal. It is likely that we need some conditions on the asymptotic behavior of harmonic four forms, like $L^{2}$ normalizability. Quite recently a general theory of $L^{2}$-harmonic forms on (non-compact) gravitational instantons is developed in [14. It is an interesting problem to explore the relation of $L^{2}$-normalizable harmonic forms and deformations of non-compact Ricci-flat metrics from mathematically more general view points.

This paper is organized as follows; throughout the paper we consider ALC metrics of $\operatorname{Spin}(7)$ holonomy that are of cohomogeneity one with the principal orbit $S p(2) / S p(1)$ or $S U(3) / U(1)$. In section two we describe invariant forms on the principal orbit $G / K=$ $S p(2) / S p(1)$ and $S U(3) / U(1)$ as the $K$-invariant subalgebra $\Lambda_{\text {inv }}$ of the differential algebra generated by the Maurer-Cartan forms of $G$. In the case of $S U(3) / U(1), \Lambda_{\text {inv }}$ depends on the choice of $U(1)$ subalgebra in the maximal torus determined by a pair of integers $(k, \ell)$. For the special embedding with $k=\ell, \Lambda_{\text {inv }}$ becomes much larger and we 
find that as a differential algebra it coincides with that of $S p(2) / S p(1)$ model supplemented by a single non-trivial cohomology class. Due to this relation the $S U(3) / U(1)$ model with the special embedding of $U(1)$ is mostly parallel with $S p(2) / S p(1)$ model. In section three we define an infinitesimal deformation of the canonical $\operatorname{Spin}(7)$ four form as a shift by the invariant self-dual four forms. We will work out the induced change in the orthonormal frame to make the deformed four form get back into the canonical form. The deformation is classified to two types. The first type deformation leads only rescalings of the vielbein and thus maintains the diagonal metric ansatz. On the other hand the second type implies the mixing of vielbein and the corresponding metric gets more involved. In section four we derive a system of first order differential equations for the self-dual four form defining a deformation to be closed in the case of background metric of cohomogeneity one. According to the two types of deformation we call the first order system $u$-system and $v$-system, respectively. We present explicit solutions to the first order system in section five. The $L^{2}$-normalizability of the solutions is examined. Due to the qualitative difference of asymptotic behavior the condition for $L^{2}$-normalizability in ALC metrics is weaker than the corresponding AC metrics. We find that ALC metrics allow $L^{2}$-normalizable self-dual four forms, but AC metrics do not in general. By looking at power series expansion we identify the solution that deforms the radius of asymptotic circle in ALC metric. We conclude that the $L^{2}$ normalizable self-dual harmonic four form controls the change in the radius of $M$-theory circle. It would be worth mentioning that the $L^{2}$ normalizable harmonic four forms of the opposite duality also play a role in the deformation of supersymmetric background of $\operatorname{Spin}(7)$ holonomy. As is shown in 15 an anti-self-dual harmonic four form in $\mathbf{2 7}$ of the irreducible decomposition of $\mathbf{3 5} \mathbf{5}_{C}$ provides a flux for brane resolution and one can construct a supersymmetric $M 2$ brane solution [16] [17]. In summary a self-dual harmonic form gives a purely gravitational deformation, while an anti-self-dual harmonic form introduces a four form flux.

There are three appendixes at the end of paper. Appendix A provides our convention of $S U(3)$ Maurer-Cartan equations. In Appendix B we summarize a formalism of Hitchin on stable forms and metrics of exceptional holonomy, which gave a background of our work. Finally we consider the deformation of the Atiyah-Hitchin metric in Appendix $\mathrm{C}$, since it allows a description similar to our approach in this paper. But there is a

\footnotetext{
${ }^{1} \mathrm{By}$ (anti-)self-duality closed forms are automatically harmonic.
} 
crucial difference. The deformation of the Atiyah-Hitchin metric breaks the ansatz of cohomogeneity one, while the deformation of ALC metrics in this paper does not.

\section{Algebra of Invariant Forms}

The geometry of $G_{2}$ and $\operatorname{Spin}(7)$ structures is closely related to three and four forms on a manifold $M$. Recently Hitchin has shown that such a relation is described in terms of certain functionals on the space of three and four forms [18 In the case of $G_{2}$ manifolds his method has turned out to be a powerful tool (see [19] for example). The space of differential forms on $M$ is usually infinite dimensional, but when the manifold $M$ admits an action of Lie group $G$ with the principal orbit $G / K$ of codimension $n$, one can make an ansatz of the three and four forms based on the algebra of invariant forms on $G / K$ and the problem can be reduced to a finite dimensional one by homogeneity. This approach is quite general and has been employed in [20] for constructing the most general $G_{2}$ metrics with the principal orbit $S^{3} \times S^{3}$. There are two homogeneous spaces $S^{7}=S p(2) / S p(1)$ (the Hopf fibration of $S^{7}$ ) and $M_{k, \ell}:=S U(3) / U(1)_{k, \ell}$ (the AloffWallach space) that are known to allow a weak $G_{2}$ structure [21]. We can consider $\operatorname{Spin}(7)$ metrics of cohomogeneity one where one of these homogeneous spaces is served as the principal orbit of codimension one. In general the algebra of invariant differential forms on a homogeneous space $G / K$ is identified as the $K$-invariant subalgebra of the exterior algebra (freely) generated by the Maurer-Cartan one forms of $G$. Let us consider the algebra of $K$-invariant forms in each case separately.

\section{$\mathbf{2 . 1} S p(2) / S p(1)$}

As a $K=S p(1)$ module the cotangent space of $S^{7}=S p(2) / S p(1)$ is decomposed as

$$
T_{e}^{*} S^{7}=P_{1} \oplus P_{2} \oplus P_{3} \oplus P_{4}
$$

where $P_{i}(i=1,2,3)$ is one dimensional and $P_{4}$ is four dimensional. Let $\sigma_{i}(i=1,2,3)$ be a basis of $P_{i}$. They are $K$-invariant and generate another $S p(1)$ subgroup of $S p(2)$ that is commuting with $K=S p(1)$. We take a basis $\Sigma_{\mu}(\mu=0,1,2,3)$ of $P_{4}$ such that

\footnotetext{
${ }^{2} \mathrm{~A}$ brief summary of his formalism is given in Appendix B.
} 
$d s^{2}=\Sigma_{0}^{2}+\Sigma_{1}^{2}+\Sigma_{2}^{2}+\Sigma_{3}^{2}$ gives a standard (conformal) metric on the base $S^{4}$ of the $S^{3}$ Hopf fibration of $S^{7}$. The self-dual combination of $\Sigma_{\mu}$ gives $K$-invariant two forms;

$$
\begin{aligned}
& \omega_{1}=\Sigma_{0} \wedge \Sigma_{1}+\Sigma_{2} \wedge \Sigma_{3}, \\
& \omega_{2}=\Sigma_{0} \wedge \Sigma_{2}+\Sigma_{3} \wedge \Sigma_{1}, \\
& \omega_{3}=\Sigma_{0} \wedge \Sigma_{3}+\Sigma_{1} \wedge \Sigma_{2} .
\end{aligned}
$$

We note the relations

$$
\begin{aligned}
& \omega_{1} \wedge \omega_{1}=\omega_{2} \wedge \omega_{2}=\omega_{3} \wedge \omega_{3}=2 \Sigma_{0} \wedge \Sigma_{1} \wedge \Sigma_{2} \wedge \Sigma_{3} \\
& \omega_{1} \wedge \omega_{2}=\omega_{2} \wedge \omega_{3}=\omega_{3} \wedge \omega_{1}=0 .
\end{aligned}
$$

The algebra $\Lambda_{\text {inv }}$ of $K$-invariant forms is generated by $\sigma_{i}$ and $\omega_{i}$ with the relation (2.3). The exterior derivative keeps the subalgebra $\Lambda_{\text {inv }}$ and on the generators we have

$$
\begin{aligned}
& d \sigma_{i}=\epsilon_{i j k} \sigma_{j} \wedge \sigma_{k}+\omega_{i}, \\
& d \omega_{i}=2 \epsilon_{i j k} \sigma_{j} \wedge \omega_{k} .
\end{aligned}
$$

By these relations we see that the space of closed three forms in $\Lambda_{\text {inv }}$ is three dimensional and the most general exact four form is given by

$$
\begin{array}{rl}
\Phi=x_{1} & d\left(\sigma_{1} \wedge \omega_{1}\right)+x_{2} d\left(\sigma_{2} \wedge \omega_{2}\right)+x_{3} d\left(\sigma_{3} \wedge \omega_{3}\right)+2 x_{4} d\left(\sigma_{1} \wedge \sigma_{2} \wedge \sigma_{3}\right) \\
& +y_{1} d\left(\sigma_{2} \wedge \omega_{3}+\sigma_{3} \wedge \omega_{2}\right)+y_{2} d\left(\sigma_{3} \wedge \omega_{1}+\sigma_{1} \wedge \omega_{3}\right)+y_{3} d\left(\sigma_{1} \wedge \omega_{2}+\sigma_{2} \wedge \omega_{1}\right) \\
=2( & \left.x_{1}+x_{2}+x_{3}\right) \Sigma_{0} \wedge \Sigma_{1} \wedge \Sigma_{2} \wedge \Sigma_{3}+2\left(x_{4}+x_{1}-x_{2}-x_{3}\right) \omega_{1} \wedge \sigma_{2} \wedge \sigma_{3} \\
& +2\left(x_{4}-x_{1}+x_{2}-x_{3}\right) \omega_{2} \wedge \sigma_{3} \wedge \sigma_{1}+2\left(x_{4}-x_{1}-x_{2}+x_{3}\right) \omega_{3} \wedge \sigma_{1} \wedge \sigma_{2} \\
& +4 y_{1}\left(\omega_{3} \wedge \sigma_{3} \wedge \sigma_{1}+\omega_{2} \wedge \sigma_{1} \wedge \sigma_{2}\right)+4 y_{2}\left(\omega_{1} \wedge \sigma_{1} \wedge \sigma_{2}+\omega_{3} \wedge \sigma_{2} \wedge \sigma_{3}\right) \\
& +4 y_{3}\left(\omega_{2} \wedge \sigma_{2} \wedge \sigma_{3}+\omega_{1} \wedge \sigma_{3} \wedge \sigma_{1}\right) .
\end{array}
$$

\section{$2.2 S U(3) / U(1)_{k, \ell}($ generic case $k \neq \ell)$}

The subgroup $U(1)_{k, \ell}$ of the homogeneous space $M_{k, \ell}:=S U(3) / U(1)_{k, \ell}$ is represented by diag. $\left(e^{i k \theta}, e^{i \ell \theta}, e^{i m \theta}\right)$ with $k+\ell+m=0$. We use the following notation for $S U(3)$ left invariant one forms;

$$
\begin{aligned}
& E^{1}=\sigma_{1}, \quad E^{2}=\sigma_{2}, \quad E^{3}=\Sigma_{1}, \quad E^{4}=\Sigma_{2}, \\
& E^{5}=\tau_{1}, \quad E^{6}=\tau_{2}, \quad E^{7}=T_{A}, \quad E^{8}=T_{B},
\end{aligned}
$$


where $E^{8}$ corresponds to the subgroup $U(1)_{k, \ell}$. The Maurer-Cartan equations in our convention are summarized in Appendix A, where we note the relation;

$$
\begin{aligned}
& \alpha_{A}=k, \quad \beta_{A}=\ell, \quad \gamma_{A}=m, \\
& \alpha_{B}=\ell-m, \quad \beta_{B}=m-k, \quad \gamma_{B}=k-\ell .
\end{aligned}
$$

As a $U(1)_{k, \ell}$ module the cotangent space of $M_{k, \ell}$ is decomposed as

$$
T_{e}^{*} M_{k, \ell}=P_{0} \oplus P_{\ell-m} \oplus P_{m-k} \oplus P_{k-\ell}
$$

where $P_{q}$ has the $U(1)$ weight $q$. A natural basis for the above decomposition is given by $E^{7}, E^{1} \pm i E^{2}, E^{3} \pm i E^{4}, E^{5} \pm i E^{6}$ with charges $0, \pm(\ell-m), \pm(m-k), \pm(k-\ell)$, respectively. The invariant differential forms of $M_{k, \ell}$ can be identified as the subalgebra of the $U(1)$ charge free part generated by

$$
\begin{aligned}
T_{A} & =E^{7} \\
\omega_{k} & =\frac{i}{2}\left(E^{2 k-1}+i E^{2 k}\right) \wedge\left(E^{2 k-1}-i E^{2 k}\right), \\
\Omega_{1}+i \Omega_{2} & =\left(E^{1}+i E^{2}\right) \wedge\left(E^{3}+i E^{4}\right) \wedge\left(E^{5}+i E^{6}\right),
\end{aligned}
$$

with the relations

$$
\begin{aligned}
\left(T_{A}\right)^{2} & =\left(\omega_{i}\right)^{2}=\left(\Omega_{j}\right)^{2}=0 \\
\omega_{i} \wedge \Omega_{j} & =0, \quad \Omega_{1} \wedge \Omega_{2}=4 \omega_{1} \wedge \omega_{2} \wedge \omega_{3} .
\end{aligned}
$$

The exterior derivative keeps the subalgebra and on the generators we have

$$
\begin{aligned}
& d T_{A}=2 k \omega_{1}+2 \ell \omega_{2}+2 m \omega_{3}, \quad d \omega_{1}=d \omega_{2}=d \omega_{3}=\Omega_{2} \\
& d \Omega_{1}=4\left(\omega_{1} \wedge \omega_{2}+\omega_{2} \wedge \omega_{3}+\omega_{3} \wedge \omega_{1}\right), \quad d \Omega_{2}=0 .
\end{aligned}
$$

The space of closed three forms is one dimensional and the most general exact four form is given by

$$
\begin{aligned}
& \Phi= x_{1} d\left(\omega_{1} \wedge T_{A}\right)+x_{2} d\left(\omega_{2} \wedge T_{A}\right)+x_{3} d\left(\omega_{3} \wedge T_{A}\right)+x_{4} d \Omega_{1} \\
&=\left(4 x_{4}+2 \ell x_{1}+2 k x_{2}\right) \omega_{1} \wedge \omega_{2}+\left(4 x_{4}+2 m x_{2}+2 \ell x_{3}\right) \omega_{2} \wedge \omega_{3} \\
& \quad+\left(4 x_{4}+2 m x_{1}+2 k x_{3}\right) \omega_{3} \wedge \omega_{1}-\left(x_{1}+x_{2}+x_{3}\right) T_{A} \wedge \Omega_{2} .
\end{aligned}
$$




\section{$2.3 S U(3) / U(1)_{1,1}($ special case $k=\ell)$}

When $k=\ell, E^{5}$ and $E^{6}$ become $U(1)$ singlets and $E^{1} \pm i E^{2}$ and $E^{3} \pm i E^{4}$ have $U(1)$ charge \pm 3 and $\mp 3$, respectively. The space of $K$-invariant forms gets much larger and is generated by $E^{5}, E^{6}, E^{7}$ and $\omega_{1}, \omega_{2}, \tilde{\Omega}_{1}, \tilde{\Omega}_{2}$. We have introduced a $K$-invariant two form

$$
\tilde{\Omega}_{1}+i \tilde{\Omega}_{2}=\left(E^{1}+i E^{2}\right) \wedge\left(E^{3}+i E^{4}\right),
$$

which satisfies $\tilde{\Omega}_{1} \wedge \tilde{\Omega}_{1}=\tilde{\Omega}_{2} \wedge \tilde{\Omega}_{2}=2 \omega_{1} \wedge \omega_{2}$ and $\omega_{i} \wedge \tilde{\Omega}_{j}=\tilde{\Omega}_{1} \wedge \tilde{\Omega}_{2}=\omega_{1} \wedge \omega_{1}=\omega_{2} \wedge \omega_{2}=0$. The exterior derivative on the generators is given by

$$
\begin{aligned}
& d E^{5}=\tilde{\Omega}_{1}-E^{6} \wedge E^{7}, \quad d E^{6}=-\tilde{\Omega}_{2}-E^{7} \wedge E^{5}, \quad d E^{7}=2 \omega^{+}-4 E^{5} \wedge E^{6}, \\
& d \omega^{+}=2\left(\tilde{\Omega}_{2} \wedge E^{5}+\tilde{\Omega}_{1} \wedge E^{6}\right), \quad d \omega^{-}=0 \\
& d \tilde{\Omega}_{1}=-2 \omega^{+} \wedge E^{6}-\tilde{\Omega}_{2} \wedge E^{7}, \quad d \tilde{\Omega}_{2}=-2 \omega^{+} \wedge E^{5}+\tilde{\Omega}_{1} \wedge E^{7}
\end{aligned}
$$

where $\omega^{ \pm}:=\omega_{1} \pm \omega_{2}$. In contrast with previous two cases there are non-trivial cohomology class $\left[\omega^{-}\right]$with degree two and its dual with degree five. One can identify $\left[\omega^{-}\right]$with the Kähler form of $\mathbf{C P}(2)$ when the coset $S U(3) / U(1)_{1,1}$ is viewed as the total space of a principal $S O(3)$ bundle over $\mathbf{C P}(2)$. It is amusing to compare the differential algebra (2.14) with that of $S p(2) / S p(1)$. By the following mapping

$$
\begin{array}{ll}
E^{5} \rightarrow-\sigma_{1}, & E^{6} \rightarrow-\sigma_{2}, \quad E^{7} \rightarrow-2 \sigma_{3}, \\
\tilde{\Omega}_{1} \rightarrow-\omega_{1}, & \tilde{\Omega}_{2} \rightarrow \omega_{2}, \quad \omega^{+} \rightarrow-\omega_{3},
\end{array}
$$

we find exactly the same differential algebra as (2.4). Hence at the level of the differential algebra of invariant forms, $S U(3) / U(1)_{1,1}$ is obtained from $S p(2) / S p(1)$ by augmenting the non-trivial cohomology class $\left[\omega^{-}\right]$. This observation has the following meaning; the complex projective space $\mathbf{C P}(2)$ is both Kähler and quaternionic Kähler]. On the other hand the four dimensional sphere $S^{4}$ is quaternionic Kähler but non-Kähler. The triplets $\omega_{i}(i=1,2,3)$ for $S^{4}$ and $\left(\tilde{\Omega}_{1}, \tilde{\Omega}_{2}, \omega^{+}\right)$for $\mathbf{C P}(2)$ define a quaternionic Kähler structure of each manifold and as remarked above $\omega^{-}$defines a Kähler structure of $\mathbf{C P}(2)$. Note that the Kähler two form $\omega^{-}$has the opposite duality to the triplet of two forms for the quaternionic Kähler structue. This is explained from the holonomy $U(2)=U(1)_{L} \times$ $S U(2)_{R}$ of $\mathbf{C P}(2)$. Namely the Kähler two form is the curvature of the line bundle

\footnotetext{
${ }^{3}$ In four dimensions the quaternionic Kähler manifold is nothing but the self-dual Einstein manifold.
} 
associated with $U(1)_{L}$, while it is the curvature of $S O(3)_{R}=S U(2)_{R} / \mathbf{Z}_{2}$ bundle which gives the quaternionic Kähler two forms. (Since $\mathbf{C P}(2)$ is not spin, there is no lift of the $S O(3)_{R}$ bundle to $S U(2)_{R}$ bundle.)

We find the space of closed three forms is three dimensional. A basis is given by exact three forms $d \omega^{+}, d \tilde{\Omega}_{1}$ and $d \tilde{\Omega}_{2}$ in (2.14). Hence, the most general exact four form has ten parameters. Taking the above correspondence to $S p(2) / S p(1)$ model into account, let us expand an invariant three form $\phi$ in the following form;

$$
\begin{aligned}
\phi= & x_{1}\left(\tilde{\Omega}_{1} \wedge E^{5}\right)-x_{2}\left(\tilde{\Omega}_{2} \wedge E^{6}\right)+\frac{1}{2} x_{3}\left(\omega^{+} \wedge E^{7}\right)-x_{4}\left(E^{5} \wedge E^{6} \wedge E^{7}\right) \\
& +y_{1}\left(\omega^{+} \wedge E^{5}-\frac{1}{2} \tilde{\Omega}_{2} \wedge E^{7}\right)+y_{2}\left(\omega^{+} \wedge E^{6}+\frac{1}{2} \tilde{\Omega}_{1} \wedge E^{7}\right)+y_{3}\left(-\tilde{\Omega}_{2} \wedge E^{5}+\tilde{\Omega}_{1} \wedge E^{6}\right) \\
& +z_{1}\left(\omega^{-} \wedge E^{5}\right)+z_{2}\left(\omega^{-} \wedge E^{6}\right)+\frac{1}{2} z_{3}\left(\omega^{-} \wedge E^{7}\right) .
\end{aligned}
$$

Then the corresponding exact four form is

$$
\begin{aligned}
\Phi:= & d \phi \\
= & \left(x_{1}+x_{2}+x_{3}\right) \omega^{+} \wedge \omega^{+}-\left(x_{4}+x_{1}-x_{2}-x_{3}\right) \tilde{\Omega}_{1} \wedge E^{6} \wedge E^{7} \\
& +\left(x_{4}-x_{1}+x_{2}-x_{3}\right) \tilde{\Omega}_{2} \wedge E^{7} \wedge E^{5}-2\left(x_{4}-x_{1}-x_{2}+x_{3}\right) \omega^{+} \wedge E^{5} \wedge E^{6} \\
& +2 y_{1}\left(-\omega^{+} \wedge E^{7} \wedge E^{5}+2 \tilde{\Omega}_{2} \wedge E^{5} \wedge E^{6}\right)+2 y_{2}\left(-2 \tilde{\Omega}_{1} \wedge E^{5} \wedge E^{6}-\omega^{+} \wedge E^{6} \wedge E^{7}\right) \\
& +2 y_{3}\left(\tilde{\Omega}_{2} \wedge E^{6} \wedge E^{7}-\tilde{\Omega}_{1} \wedge E^{7} \wedge E^{5}\right) \\
& -z_{1}\left(\omega^{-} \wedge E^{6} \wedge E^{7}\right)-z_{2}\left(\omega^{-} \wedge E^{7} \wedge E^{5}\right)-2 z_{3}\left(\omega^{-} \wedge E^{5} \wedge E^{6}\right) .
\end{aligned}
$$

\section{Deformation of Four Form and ALC metric}

A metric with special holonomy is Ricci flat and an overall scaling of the metric always gives one dimensional trivial modulus of Ricci flat metrics. When a Ricci-flat metric of cohomogeneity one is obtained as a resolution of the cone metric over $G / K$, this scale parameter controls the resolution of conical singularity and it is related to the volume of the bolt singularity; $S^{4}$ for $S p(2) / S p(1)$ case and $\mathbf{C P}^{2}$ for $S U(3) / U(1)$ case. Hence we can fix the modulus of scaling by normalizing the size of the bolt singularity. Then non-trivial moduli come from deformations of the traceless part of metric. When the manifold is compact, infinitesimal deformations that keep Ricci-flatness are given by zero modes of the Lichnerowicz laplacian [13]. A reduction of the holonomy to special 
holonomy allows us to identify these zero modes with zero-modes of exterior derivative on differential form (harmonic forms) of an appropriate degree. For example for $G_{2}$ holonomy the formal dimensions of $G_{2}$ metrics is given by the third Betti number $b_{3}$ and for $\operatorname{Spin}(7)$ the dimensions is given by $1+b_{4}^{+}$where $b_{4}^{+}$is the Betti number of self-dual four forms [12]. For non-compact manifold the correspondence of infinitesimal deformations of Ricci flat metrics and the harmonic forms becomes subtle. We may impose some conditions on normalizablity and/or asymptotic behavior on differential forms. The condition we should impose is not necessarily unique and it would characterize the physical meaning of corresponding moduli. For example the moduli of metrics that come from $L^{2}$-normalizable closed forms will not change the asymptotic behavior and are expected to be dynamical, while those from $L^{2}$-non-normalizable forms are nondynamical. They can change the asymptotic behavior of the metric and are regarded as a change of background metric. In the following based on the coset geometry of $S p(2) / S p(1)$ and $S U(3) / U(1)$, we will see quite explicitly the correspondence between the harmonic self-dual four forms and infinitesimal deformations of $\operatorname{Spin}(7)$ metric. In our models of $S p(2) / S p(1)$ and $S U(3) / U(1)$ the fourth de Rham cohomology of the coset is trivial and we will take exact four forms on the coset to produce self-dual four forms on the total space. Thus the harmonic $L^{2}$ normalizable four forms $G$ in this paper is exact $G=d C$ in algebraic sense. However, it is non-trivial in the sense of $L^{2}$-cohomology, since the three form potential $C$ is not $L^{2}$ normalizable in general.

\section{1 $S p(2) / S p(1)$}

Assume that the vielbeins are given by

$$
\begin{aligned}
& e^{1}=a(t) \Sigma_{1}, \quad e^{3}=a(t) \Sigma_{2}, \quad e^{5}=a(t) \Sigma_{3}, \quad e^{7}=a(t) \Sigma_{0} \\
& e^{2}=b_{1}(t) \sigma_{1}, \quad e^{4}=b_{2}(t) \sigma_{2}, \quad e^{6}=b_{3}(t) \sigma_{3} .
\end{aligned}
$$

As the canonical ASD four form of $\operatorname{Spin}(7)$ structure on $\mathbf{R} \times S p(2) / S p(1)$, we take

$$
\Omega_{0}^{\mathrm{ASD}}=d t \wedge * \rho_{0}-\rho_{0}
$$

\footnotetext{
${ }^{4}$ Our convention is that the canonical four form of $\operatorname{Spin}(7)$ structure is a closed anti-self-dual four form. If it is self-dual, $b_{4}^{+}$should be replaced by $b_{4}^{-}$; the Betti number of anti-self-dual four forms.
} 
where

$$
\begin{aligned}
\rho_{0}= & -e^{1357}+e^{1467}+e^{3456}+e^{2367}+e^{1256}+e^{2457}+e^{1234}, \\
= & \frac{1}{2}\left(e^{12}+e^{34}+e^{56}\right)^{2}-\operatorname{Re}\left[\left(e^{1}+i e^{2}\right)\left(e^{3}+i e^{4}\right)\left(e^{5}+i e^{6}\right)\right] \wedge e^{7}, \\
= & a^{4} \Sigma_{0} \wedge \Sigma_{1} \wedge \Sigma_{2} \wedge \Sigma_{3}-a^{2} b_{2} b_{3}\left(\omega_{1} \wedge \sigma_{2} \wedge \sigma_{3}\right)-a^{2} b_{3} b_{1}\left(\omega_{2} \wedge \sigma_{3} \wedge \sigma_{1}\right) \\
& \quad-a^{2} b_{1} b_{2}\left(\omega_{3} \wedge \sigma_{1} \wedge \sigma_{2}\right),
\end{aligned}
$$

and

$$
\begin{aligned}
* \rho_{0} & =-e^{246}+e^{352}+e^{712}+e^{514}+e^{734}+e^{136}+e^{756} \\
& =\left(e^{12}+e^{34}+e^{56}\right) \wedge e^{7}+\operatorname{Im}\left[\left(e^{1}+i e^{2}\right)\left(e^{3}+i e^{4}\right)\left(e^{5}+i e^{6}\right)\right] \\
& =-b_{1} b_{2} b_{3}\left(\sigma_{1} \wedge \sigma_{2} \wedge \sigma_{3}\right)+a^{2} b_{1}\left(\omega_{1} \wedge \sigma_{1}\right)+a^{2} b_{2}\left(\omega_{2} \wedge \sigma_{2}\right)+a^{2} b_{3}\left(\omega_{3} \wedge \sigma_{3}\right) .
\end{aligned}
$$

The Hodge operator $*$ is defined by the coset metric $\hat{g}(t)=e^{a} \otimes e^{a}$ and we have $\alpha \wedge * \alpha=$ $e^{1234567}$ if $\alpha$ is an exterior product of $e^{a}$. When the four form $\Omega_{0}^{\mathrm{ASD}}$ is closed, the $\operatorname{Spin}(7)$ structure is called torsion free and the corresponding metric $g=d t^{2}+\hat{g}(t)$ has $\operatorname{Spin}(7)$ holonomy. It is easy to see that the octonionic instanton equation for the spin connection implies $d \Omega_{0}^{\mathrm{ASD}}=0$.

Now let us look at an infinitesimal shift of the canonical four form by a self-dual four form

$$
\begin{aligned}
\Omega & =\Omega_{0}^{\mathrm{ASD}}+\epsilon G^{\mathrm{SD}} \\
& =\left(d t \wedge * \rho_{0}-\rho_{0}\right)+\epsilon(d t \wedge \Psi+\Phi) \\
& =d t \wedge \sigma-\rho,
\end{aligned}
$$

where $\sigma \equiv * \rho_{0}+\epsilon \Psi$ is a three form and $\rho \equiv \rho_{0}-\epsilon \Phi$ is a four form. The seven dimensional duality $* \Phi=\Psi$ implies the eight dimensional self-duality of $G^{\mathrm{SD}}=d t \wedge \Psi+\Phi$. As before for the deformed four form $\Omega$ to define a torsion free $\operatorname{Spin}(7)$ structure we must require that $d \Omega=0$. Let us take the invariant four form $\Phi$ that is closed in seven dimensional sense. Hence in the $S p(2) / S p(1)$ model we take (see Eq.(2.5));

$$
\begin{aligned}
\Phi=u_{1} a^{4}\left(\Sigma_{0} \wedge \Sigma_{1} \wedge \Sigma_{2} \wedge \Sigma_{3}\right)+u_{2} a^{2} b_{2} b_{3}\left(\omega_{1} \wedge \sigma_{2} \wedge \sigma_{3}\right) \\
+u_{3} a^{2} b_{3} b_{1}\left(\omega_{2} \wedge \sigma_{3} \wedge \sigma_{1}\right)+u_{4} a^{2} b_{1} b_{2}\left(\omega_{3} \wedge \sigma_{1} \wedge \sigma_{2}\right) \\
+v_{1}\left(\omega_{2} \wedge \sigma_{2} \wedge \sigma_{3}+\omega_{1} \wedge \sigma_{3} \wedge \sigma_{1}\right)+v_{2}\left(\omega_{3} \wedge \sigma_{3} \wedge \sigma_{1}+\omega_{2} \wedge \sigma_{1} \wedge \sigma_{2}\right) \\
\quad+v_{3}\left(\omega_{1} \wedge \sigma_{1} \wedge \sigma_{2}+\omega_{3} \wedge \sigma_{2} \wedge \sigma_{3}\right)
\end{aligned}
$$


In our ansatz of cohomogeneity one metric the coefficients $u_{i}$ and $v_{i}$ are functions of $t$ and $d \Phi=d t \wedge(d \Phi / d t)$ in eight dimensions. $d G^{\mathrm{SD}}=0$ means $d \Phi / d t=d * \Phi$. This condition gives a first order system for the coefficients $u_{i}$ and $v_{i}$. We will describe it more explicitly in the next section.

When $v_{i}=0$ the diagonal metric ansatz is maintained. In fact assuming that new vielbeins are

$$
\tilde{e}^{i}=\left(1+\epsilon U_{i}\right) e^{i}, \quad U_{1}=U_{3}=U_{5}=U_{7}
$$

we obtain in the leading order

$$
\begin{aligned}
& U_{1}=\frac{1}{4} u_{1}, \quad U_{2}=\frac{1}{2}\left(-u_{2}+u_{3}+u_{4}\right)-\frac{1}{4} u_{1}, \\
& U_{4}=\frac{1}{2}\left(u_{2}-u_{3}+u_{4}\right)-\frac{1}{4} u_{1}, \quad U_{6}=\frac{1}{2}\left(u_{2}+u_{3}-u_{4}\right)-\frac{1}{4} u_{1} .
\end{aligned}
$$

together with a redefinition of "time" variable

$$
d \tilde{t}=\left(1-\epsilon\left(4 U_{1}+U_{2}+U_{4}+U_{6}\right)\right) d t
$$

We can see that $\Omega$ is transformed into the canonical form in terms of these new vielbeins.

On the other hand non-vanishing $v_{i}$ causes a "mixing" of the original vielbein. For example one can consider the deformation by

$$
\begin{aligned}
\Phi_{\text {mix }}= & v_{1}\left(b_{1}\left(e^{7346}+e^{5146}\right)+b_{2}\left(e^{7162}+e^{3562}\right)\right) \\
& +v_{2}\left(b_{2}\left(e^{7562}+e^{1362}\right)+b_{3}\left(e^{7324}+e^{5124}\right)\right) \\
& +v_{3}\left(b_{3}\left(e^{7124}+e^{3524}\right)+b_{1}\left(e^{7546}+e^{1346}\right)\right)
\end{aligned}
$$

where we have scaled the infinitesimal deformation parameters $v_{i}$. We have checked that the deformed four form can be transformed into the canonical form by allowing the "mixing" of the orthonormal frame. Namely we find a solution to the condition

$$
\Omega_{0}^{A S D}+\epsilon\left(\Phi_{m i x}+d t \wedge * \Phi_{m i x}\right)=d t \wedge \tilde{*} \tilde{\rho}_{0}-\tilde{\rho}_{0}
$$

where $\tilde{\rho}_{0}$ and $\tilde{*} \tilde{\rho}_{0}$ are given by (3.3) and (3.4) with $e^{i}$ being replaced by a new orthonormal frame $\tilde{e}^{i}$. The new orthonormal frame $\tilde{e}^{i}$ is assumed to be a linear combination of the original vielbein. Furthermore one is forced to change the frame of the radial direction $e^{0}=d t$ mixed with the frames $e^{i}$ of the principal orbit. Due to this mixing of the radial geodesic direction and the principal orbit, it is not clear if the deformed metric remains of 
cohomogeneity one. As we will see later in explicit examples there are no $L^{2}$-normalizable solution providing the deformations of this type (see the $v$-system in the next section) and we will leave this issue as an open problem.

\section{$3.2 S U(3) / U(1)_{1,1}($ special case $k=\ell)$}

In this case we take the following vielbeins given by

$$
\begin{aligned}
& e^{1}=a(t) E^{1}, \quad e^{2}=a(t) E^{2}, \quad e^{3}=b(t) E^{3}, \quad e^{4}=b(t) E^{4}, \\
& e^{5}=c_{1}(t) E^{5}, \quad e^{6}=c_{2}(t) E^{6}, \quad e^{7}=f(t) E^{7} .
\end{aligned}
$$

and the canonical ASD four form of $\operatorname{Spin}(7)$ structure on $\mathbf{R} \times S U(3) / U(1)_{1,1}$

$$
\Omega_{0}^{\mathrm{ASD}}=d t \wedge * \rho_{0}-\rho_{0}
$$

where

$$
\begin{aligned}
\rho_{0}= & e^{1234}+e^{1256}+e^{3456}-e^{1367}+e^{2467}-e^{1457}-e^{2357} \\
= & \frac{1}{2}\left(e^{12}+e^{34}+e^{56}\right)^{2}-\operatorname{Im}\left[\left(e^{1}+i e^{2}\right)\left(e^{3}+i e^{4}\right)\left(e^{5}+i e^{6}\right)\right] \wedge e^{7}, \\
= & \frac{1}{2} a^{2} b^{2}\left(\omega^{+} \wedge \omega^{+}\right)+\frac{1}{2}\left(a^{2}+b^{2}\right) c_{1} c_{2}\left(\omega^{+} \wedge E^{5} \wedge E^{6}\right)+\frac{1}{2}\left(a^{2}-b^{2}\right) c_{1} c_{2}\left(\omega^{-} \wedge E^{5} \wedge E^{6}\right) \\
& \quad+a b c_{2} f\left(-\tilde{\Omega}_{1} \wedge E^{6} \wedge E^{7}\right)+a b c_{1} f\left(\tilde{\Omega}_{2} \wedge E^{7} \wedge E^{5}\right)
\end{aligned}
$$

and

$$
\begin{aligned}
* \rho_{0}= & e^{567}+e^{347}+e^{127}+e^{315}+e^{524}+e^{461}+e^{362} \\
= & \left(e^{12}+e^{34}+e^{56}\right) \wedge e^{7}-\operatorname{Re}\left[\left(e^{1}+i e^{2}\right)\left(e^{3}+i e^{4}\right)\left(e^{5}+i e^{6}\right)\right], \\
= & c_{1} c_{2} f\left(E^{5} \wedge E^{6} \wedge E^{7}\right)+\frac{1}{2}\left(a^{2}+b^{2}\right) f\left(\omega_{+} \wedge E^{7}\right)+\frac{1}{2}\left(a^{2}-b^{2}\right) f\left(\omega_{-} \wedge E^{7}\right) \\
& \quad+a b c_{1}\left(-\tilde{\Omega}_{1} \wedge E^{5}\right)+a b c_{2}\left(\tilde{\Omega}_{2} \wedge E^{6}\right) .
\end{aligned}
$$

An infinitesimal deformation of $\operatorname{Spin}(7)$ four form is defined by considering the following most general ansatz of the invariant four form $\Phi$;

$$
\begin{aligned}
\Phi & =u_{1}(t) e^{1234}+u_{2}(t) e^{1256}+u_{3}(t) e^{3456}+u_{4}(t)\left(-e^{1367}+e^{2467}\right)+u_{5}(t)\left(-e^{2357}-e^{1457}\right) \\
& +v_{1}(t) e^{1267}-v_{2}(t) e^{1257}+v_{3}(t) e^{3467}-v_{4}(t) e^{3457}+v_{5}(t)\left(e^{2457}-e^{1357}\right) \\
& +\ell_{1}(t)\left(-e^{2456}+e^{1356}\right)+\ell_{2}(t)\left(e^{2356}+e^{1456}\right)+\ell_{3}(t)\left(e^{2367}+e^{1467}\right) .
\end{aligned}
$$


We can check that if we impose the condition

$$
\ell_{1}=\frac{f}{a b c_{1}}\left(a^{2} v_{1}+b^{2} v_{3}\right), \quad \ell_{2}=-\frac{f}{a b c_{2}}\left(a^{2} v_{2}+b^{2} v_{4}\right), \quad \ell_{3}=-\frac{c_{1}}{c_{2}} v_{5},
$$

then $\Phi$ is closed form on $S U(3) / U(1)$ and expressed as follows (see Eq.(2.17));

$$
\begin{aligned}
\Phi & =\frac{1}{2} u_{1} a^{2} b^{2}\left(\omega^{+} \wedge \omega^{+}\right)-u_{4} a b c_{2} f\left(\tilde{\Omega}_{1} \wedge E^{6} \wedge E^{7}\right)+u_{5} a b c_{1} f\left(\tilde{\Omega}_{2} \wedge E^{7} \wedge E^{5}\right) \\
& +\frac{1}{2}\left(u_{2} a^{2}+u_{3} b^{2}\right) c_{1} c_{2}\left(\omega^{+} \wedge E^{5} \wedge E^{6}\right)+a b c_{1} f v_{5}\left(\tilde{\Omega}_{1} \wedge E^{7} \wedge E^{5}-\tilde{\Omega}_{2} \wedge E^{5} \wedge E^{6}\right) \\
& +c_{1} f\left(v_{2} a^{2}+v_{4} b^{2}\right)\left(\frac{1}{2} \omega^{+} \wedge E^{7} \wedge E^{5}-\tilde{\Omega}_{2} \wedge E^{5} \wedge E^{6}\right) \\
& +c_{2} f\left(v_{1} a^{2}+v_{3} b^{2}\right)\left(\frac{1}{2} \omega^{+} \wedge E^{6} \wedge E^{7}+\tilde{\Omega}_{1} \wedge E^{5} \wedge E^{6}\right) \\
& +\frac{1}{2}\left(u_{2} a^{2}-u_{3} b^{2}\right) c_{1} c_{2} \omega^{-} \wedge E^{5} \wedge E^{6}+\frac{1}{2}\left(v_{1} a^{2}-v_{3} b^{2}\right) c_{2} f \omega^{-} \wedge E^{6} \wedge E^{7} \\
& +\frac{1}{2}\left(v_{2} a^{2}-v_{4} b^{2}\right) c_{1} f \omega^{-} \wedge E^{7} \wedge E^{5} .
\end{aligned}
$$

We find the parameters $u_{i}$ give rise to a diagonal deformation of the metric $\tilde{g}=g+$ $\epsilon h_{\text {diag }} / 2(\epsilon \ll 1)$. By using the orthonormal basis $g=d t^{2}+e^{a} \otimes e^{a}$, the metric is written as

$$
h_{\text {diag }}=h_{0} d t^{2}+\sum_{a=1}^{7} h_{a} e^{a} \otimes e^{a},
$$

where

$$
\begin{aligned}
& h_{0}=u_{1}+u_{2}+u_{3}+2\left(u_{4}+u_{5}\right), \quad h_{1}=h_{2}=-u_{1}-u_{2}+u_{3}, \\
& h_{3}=h_{4}=-u_{1}+u_{2}-u_{3}, \quad h_{5}=u_{1}-u_{2}-u_{3}+2\left(u_{4}-u_{5}\right), \\
& h_{6}=u_{1}-u_{2}-u_{3}-2\left(u_{4}-u_{5}\right), \quad h_{7}=u_{1}+u_{2}+u_{3}-2\left(u_{4}+u_{5}\right) .
\end{aligned}
$$

We note that by the correspondence $u_{1} \rightarrow u_{1}, u_{2}=u_{3} \rightarrow u_{4}, u_{4} \rightarrow u_{2}, u_{5} \rightarrow u_{3}$ the result of $S p$ model is reproduced up to an overall factor.

\section{First Order System for Harmonic Four Forms}

In this section we will give the condition for the self-dual four form $G^{S D}$ to be closed and hence to be harmonic. When an eight-manifold $(M, g)$ is of cohomogeneity one, that is, $M$ admits an action of the Lie group $G$ with seven dimensional principal orbits $G / K$, 
the manifold is locally $M \simeq \mathbf{R} \times G / K$. Taking a unit vector field normal to the orbit, we can write the metric in the form

$$
g=d t^{2}+\hat{g}(t)
$$

where $\hat{g}(t)$ is a $G$-invariant metric on the orbit $G / K$. With this metric the condition for $G^{S D}$ is expressed by a system of first order differential equations for the coefficients of invariant form on $G / K$.

\section{1 $S U(3) / U(1)_{1,1}$}

Our choice of the vielbeins (3.12) implies the following diagonal form of $\hat{g}(t)$ for all $t$;

$$
\begin{aligned}
\hat{g}(t)= & a(t)^{2}\left(E^{1} \otimes E^{1}+E^{2} \otimes E^{2}\right)+b(t)^{2}\left(E^{3} \otimes E^{3}+E^{4} \otimes E^{4}\right) \\
& +c_{1}(t)^{2} E^{5} \otimes E^{5}+c_{2}(t)^{2} E^{6} \otimes E^{6}+f(t)^{2} E^{7} \otimes E^{7}
\end{aligned}
$$

Let us consider a self-dual 4-form on $M$ of the following form;

$$
G^{S D}=\Phi+e^{0} \wedge * \Phi, \quad\left(e^{0}:=d t\right)
$$

where $\Phi$ is expanded by a basis for exact invariant four-forms on $G / K$ and an explicit form is given by (3.18). Since $\Phi$ in (4.3) is exact on $G / K$, the closeness condition $d G^{S D}=0$ is expressed by

$$
\frac{d}{d t} \Phi=d * \Phi
$$

From the result in section 2.3 we see that $* \Phi$ is a $K$-invariant three form and $d * \Phi$ can be expanded by the basis for exact four forms given in section 2.3. Thus, (4.4) yields the following first order differential equations;

(A) $u$-system

$$
\begin{aligned}
& \frac{d}{d t}\left(a^{2} b^{2} u_{1}\right)-2 a^{2} f u_{3}-2 b^{2} f u_{2}+2 a b c_{1} u_{4}+2 a b c_{2} u_{5}=0 \\
& \frac{d}{d t}\left(a^{2} c_{1} c_{2} u_{2}\right)+4 a^{2} f u_{3}-2 c_{1} c_{2} f u_{1}+2 a b c_{1} u_{4}+2 a b c_{2} u_{5}=0 \\
& \frac{d}{d t}\left(b^{2} c_{1} c_{2} u_{3}\right)+4 b^{2} f u_{2}-2 c_{1} c_{2} f u_{1}+2 a b c_{1} u_{4}+2 a b c_{2} u_{5}=0 \\
& \frac{d}{d t}\left(a b c_{2} f u_{4}+a b c_{1} f u_{5}\right)+2 f\left(a^{2} u_{3}+b^{2} u_{2}+c_{1} c_{2} u_{1}\right)=0 \\
& \frac{d}{d t}\left(a b c_{2} f u_{4}-a b c_{1} f u_{5}\right)+2\left(a b c_{1} u_{4}-a b c_{2} u_{5}\right)=0
\end{aligned}
$$


(B) $v$-system

$$
\begin{aligned}
& \frac{d}{d t}\left(a^{2} c_{2} f v_{1}\right)+\left(a^{2} c_{1}+2 b^{2} f^{2} / c_{1}\right) v_{3}+\left(2 a^{2} f^{2} / c_{1}\right) v_{1}=0 \\
& \frac{d}{d t}\left(b^{2} c_{2} f v_{3}\right)+\left(b^{2} c_{1}+2 a^{2} f^{2} / c_{1}\right) v_{1}+\left(2 b^{2} f^{2} / c_{1}\right) v_{3}=0 \\
& \frac{d}{d t}\left(a^{2} c_{1} f v_{2}\right)+\left(a^{2} c_{2}+2 b^{2} f^{2} / c_{2}\right) v_{4}+\left(2 a^{2} f^{2} / c_{2}\right) v_{2}=0 \\
& \frac{d}{d t}\left(b^{2} c_{1} f v_{4}\right)+\left(b^{2} c_{2}+2 a^{2} f^{2} / c_{2}\right) v_{2}+\left(2 b^{2} f^{2} / c_{2}\right) v_{4}=0 \\
& \frac{d}{d t}\left(a b c_{1} f v_{5}\right)+\frac{a b}{c_{2}}\left(c_{1}^{2}+c_{2}^{2}\right) v_{5}=0
\end{aligned}
$$

We note that the first order system for $u_{i}$ decouples from that for $v_{i}$. Furthermore the $v$-system decomposes into three independent systems for $\left(v_{1}, v_{3}\right),\left(v_{2}, v_{4}\right)$ and $v_{5}$. The $\left(v_{2}, v_{4}\right)$ system is obtained from the $\left(v_{1}, v_{3}\right)$ system by the replacement $c_{2} \rightarrow c_{1}$.

\section{$4.2 S U(3) / U(1)_{k, \ell}(k \neq \ell)$}

The $u$-system in the generic case $(k \neq \ell)$ can be obtained similarly. The invariant metric $\hat{g}$ on the coset is given by (4.2) with $c \equiv c_{1}=c_{2}$ and according to the result in section 2.2 , we put $u_{4}=u_{5}, v_{i}=\ell_{j}=0$ in the expansion of $G^{S D}$ in terms of invariant forms. Then the $u$-system is reduced to

$$
\begin{aligned}
& \frac{d}{d t}\left(a^{2} b^{2} u_{1}\right)=-4 a b c u_{4}+2 k f b^{2} u_{2}+2 \ell f a^{2} u_{3} \\
& \frac{d}{d t}\left(a^{2} c^{2} u_{2}\right)=-4 a b c u_{4}+2 k f c^{2} u_{1}+2 m f a^{2} u_{3} \\
& \frac{d}{d t}\left(b^{2} c^{2} u_{3}\right)=-4 a b c u_{4}+2 \ell f c^{2} u_{1}+2 m f b^{2} u_{2} \\
& \frac{d}{d t}\left(a b c f u_{4}\right)=-f\left(a^{2} u_{3}+b^{2} u_{2}+c^{2} u_{1}\right)
\end{aligned}
$$

This equation has been derived in [5], but the overall sign of the right-hand side is reversed here. The sign is determined by the duality of closed four forms. In [5] we were interested in the anti-self-dual closed four forms (in our present convention円) in order to construct supersymmetric brane solutions following [22] 16] [1]. When the canonical $\operatorname{Spin}(7)$ form $\Omega$ is taken to be anti-self-dual, it is self-dual harmonic four forms that is relevant to

\footnotetext{
${ }^{5}$ Unfortunately the convention of [5], which is the same as [22], is opposite to the present paper and 16.
} 
metric deformations. On the other hand anti-self-dual harmonic four forms are used to construct brane solutions that preserve supersymmetry. We also note that the equation for the reversed duality satisfied the linear relation $u_{1}+u_{2}+u_{3}+4 u_{4}=$ const, while there is no such a relation in (4.7).

\section{3 $S p(2) / S p(1)$}

Using the correspondence (2.15) found in section two, we can derive the first order system for $S p(2) / S p(1)$ model rather easily. Recall that the metric ansatz is

$$
g=d t^{2}+a(t)^{2}\left(\Sigma_{0}^{2}+\Sigma_{1}^{2}+\Sigma_{2}^{2}+\Sigma_{3}^{2}\right)+b_{1}(t)^{2} \sigma_{1}^{2}+b_{2}(t)^{2} \sigma_{2}^{2}+b_{3}(t)^{2} \sigma_{3}^{2} .
$$

Let us begin with the $u$-system. By substituting $a=b \rightarrow a, c_{1} \rightarrow b_{1} c_{2} \rightarrow b_{2},-2 f \rightarrow b_{3}$ and making the replacement $u_{1} \rightarrow u_{1}, u_{2}=u_{3} \rightarrow u_{4}, u_{4} \rightarrow u_{2}, u_{5} \rightarrow u_{3}$ we obtain

(A) $u$-system

$$
\begin{aligned}
& \frac{d}{d t}\left(a^{4} u_{1}\right)+2 a^{2}\left(b_{1} u_{2}+b_{2} u_{3}+b_{3} u_{4}\right)=0, \\
& \frac{d}{d t}\left(a^{2} b_{2} b_{3} u_{2}\right)+b_{1} b_{2} b_{3} u_{1}+2 a^{2}\left(-b_{1} u_{2}+b_{2} u_{3}+b_{3} u_{4}\right)=0 \\
& \frac{d}{d t}\left(a^{2} b_{3} b_{1} u_{3}\right)+b_{1} b_{2} b_{3} u_{1}+2 a^{2}\left(b_{1} u_{2}-b_{2} u_{3}+b_{3} u_{4}\right)=0 \\
& \frac{d}{d t}\left(a^{2} b_{1} b_{2} u_{4}\right)+b_{1} b_{2} b_{3} u_{1}+2 a^{2}\left(b_{1} u_{2}+b_{2} u_{3}-b_{3} u_{4}\right)=0 .
\end{aligned}
$$

The $u$-system for $S p(2) / S p(1)$ model becomes more symmetric in the sense that it has a cyclic symmetry in $\left(u_{2}, u_{3}, u_{4}\right)$.

In the same way we can obtain the following $\tilde{v}$-system for $S p(2) / S p(1)$

(B) $\tilde{v}$-system

$$
\begin{aligned}
& \frac{d}{d t}\left(a^{2} b_{2} b_{3} \tilde{v}_{1}\right)-\frac{2 a^{2}}{b_{1}}\left(b_{3}^{2}+b_{1}^{2}\right) \tilde{v}_{1}=0, \\
& \frac{d}{d t}\left(a^{2} b_{3} b_{1} \tilde{v}_{2}\right)-\frac{2 a^{2}}{b_{2}}\left(b_{1}^{2}+b_{2}^{2}\right) \tilde{v}_{2}=0, \\
& \frac{d}{d t}\left(a^{2} b_{1} b_{2} \tilde{v}_{3}\right)-\frac{2 a^{2}}{b_{3}}\left(b_{2}^{2}+b_{3}^{2}\right) \tilde{v}_{3}=0,
\end{aligned}
$$

where we have made the identification $\tilde{v}_{1} \equiv v_{1}=v_{3}, \tilde{v}_{2} \equiv v_{5}$ and $\tilde{v}_{3} \equiv\left(b_{2} / b_{3}\right) v_{2}=$ $\left(b_{2} / b_{3}\right) v_{4}$. Note that the $\tilde{v}$ system decouples completely into three independent ODE and again we find a cyclic symmetry in $\left(\tilde{v}_{1}, \tilde{v}_{2}, \tilde{v}_{3}\right)$. 


\section{Examples}

To solve the $u$-system and the $v$-system obtained in the last section we need an expression of the background metric $a(t), b(t), c_{i}(t)$ and $f(t)$. Let us consider a few examples of explicit ALC metrics. We also discuss AC metrics briefly, since qualitative features are different.

\section{ALC metric}

The first example is a deformation of the ALC $\operatorname{Spin}(7)$ metric $\mathbf{B}_{8}$ on the bundle of chiral spinors over $S^{4}$. In this example the existence of deformation was established globally in [1], as a family of complete metrics $\mathbf{B}_{8}^{ \pm}$is known explicitly. Our calculation shows that the infinitesimal variation of the metric is controlled by an $L^{2}$-normalizable harmonic self-dual four form $G^{S D}$. The second example is a deformation of the ALC $\operatorname{Spin}(7)$ metric on the $\operatorname{Spin}^{c}$ bundle over $\mathbf{C P}(2)$ [3] [5] [n this case a non-trivial deformation was suggested by numerical analyses [3] [5] and the existence of an $L^{2}$-normalizable $G^{S D}$ gives a further evidence for the deformation.

Let us start with the $\mathbf{B}_{8}$ metric. The metric is given by (4.8) with

$$
a^{2}=r^{2}-\ell^{2}, \quad b_{1}^{2}=b_{2}^{2}=(r-3 \ell)(r+\ell), \quad b_{3}^{2}=4 \ell^{2}(r-3 \ell)(r+\ell) /(r-\ell)^{2}
$$

and $d t=(r-\ell) / \sqrt{(r-3 \ell)(r+\ell)} d r$. The radial coordinate $r$ runs from the singular orbit $S^{4}$ at $r=3 \ell$ to infinity. From the $u$-system (4.9) with $b_{1}=b_{2}$, it is easy to find

$$
u_{2}-u_{3}=k_{0} \frac{(r-3 \ell)^{3}}{(r+\ell)^{6}} \exp (2 r / \ell)
$$

with a constant $k_{0}$. Since this solution has exponential growth at infinity, we will take $k_{0}=0$. A convenient way to obtain the remaining solutions is to introduce a new variable $f=a^{2} b_{1} b_{2}\left(u_{2}+u_{3}\right)$. Taking derivatives of the first-order equations (4.9), we obtain the following differential equation of Fuchsian type after rescaling the radial coordinate $x=r / \ell$;

$$
\frac{d^{3} f}{d x^{3}}+p_{1}(x) \frac{d^{2} f}{d x^{2}}+p_{2}(x) \frac{d f}{d x}+p_{3}(x) f=0
$$

where

$$
p_{1}=\frac{2\left(3 x^{2}-10 x-1\right)}{(x+1)(x-1)(x-3)},
$$




$$
\begin{aligned}
& p_{2}=-\frac{6 x^{3}-18 x^{2}+58 x-110}{(x+1)^{2}(x-1)(x-3)^{2}} \\
& p_{3}=-\frac{8\left(3 x^{4}-24 x^{3}+46 x^{2}-32 x-9\right)}{(x+1)^{3}(x-1)(x-3)^{3}} .
\end{aligned}
$$

This equation can be integrated and we find three linearly independent solutions

$$
\begin{aligned}
f_{1} & =\frac{64(x-3)^{3}}{(x+1)^{2}(x-1)^{2}}, \quad f_{2}=\frac{3 x-5}{(x+1)^{2}(x-1)^{2}(x-3)}, \\
f_{3} & =\frac{(x-3)^{3}\left(3 x^{4}+24 x^{3}+98 x^{2}+288 x+723\right)}{2(x+1)^{2}(x-1)^{2}} .
\end{aligned}
$$

The second solution $f_{2}$ is singular at the point $x=3$ which corresponds to the singular orbit $S^{4}$ and hence the regular solution in the region $x \geq 3$ is a linear combination $f(x)=$ $k_{1} f_{1}(x)+k_{3} f_{3}(x)$. The remaining functions $u_{1}$ and $u_{4}$ are given by taking derivatives of $f(x)$.

In the region near the singular orbit the local deformation of the $\mathbf{B}_{8}$ metric is characterized by two parameters, which we shall label as $m$ and $q$ [3] [5]. The lower order terms near the singular orbit are

$$
\begin{aligned}
a(t) & =m\left(1+\frac{3}{4}(t / m)^{2}+\cdots\right), \\
b_{1}(t) & =b_{2}(t)=t\left(1-q(t / m)^{2}+\cdots\right), \\
b_{3}(t) & =t\left(1+(2 q-1)(t / m)^{2}+\cdots\right) .
\end{aligned}
$$

The $\mathbf{B}_{8}$ metric corresponds to $m=2 \sqrt{2} \ell$ and $q=0$. As we have seen in Section 3, the $u$-system gives rise to a diagonal deformation and the solutions $f(x)$ and (5.2) with $k_{0}=0$ induces the following transformation,

$$
q=0 \rightarrow \tilde{q}=-5\left(k_{1}-28 k_{3}\right) / 32, \quad m=2 \sqrt{2} \ell \rightarrow \tilde{m}=2 \sqrt{2} \ell\left(1+3\left(k_{1}-35 k_{3}\right) / 16\right) .
$$

The four-form $G^{S D}$ is not $L^{2}$-normalizable, unless we do not impose the condition $k_{3}=0$ in the solution $f(x)$. When $k_{3}=0$ the solution explicitly becomes

$$
\begin{aligned}
& u_{1}=-\frac{16 k_{1}\left(5 x^{3}-9 x^{2}+15 x-3\right)}{(x+1)^{4}(x-1)^{3}}, \quad u_{2}=u_{3}=\frac{32 k_{1}(x-3)}{(x+1)^{4}(x-1)^{2}}, \\
& u_{4}=\frac{8 k_{1}(x-3)\left(5 x^{2}-2 x+1\right)}{(x+1)^{4}(x-1)^{3}}
\end{aligned}
$$


which gives an $L^{2}$-normalizable $G^{S D}$. The $L^{2}$-normalizable solution leads to a correlation in transformations of the two parameters $m$ and $q$. However, we note that the transformation of $m$ can be absorbed in an overall scaling of the metric (a trivial deformation). Hence we conclude that the deformation of $\mathbf{B}_{8}$ that changes the asymptotic radius of the $S^{1}$ fiber is essentially controlled by the $L^{2}$-normalizable $G^{S D}$. We should mention that this $L^{2}$-normalizable solution was obtained in [1], where it has been noticed the ALC metric $\mathbf{B}_{8}$ admits a unique $L^{2}$-normalizable harmonic four form of each duality. In [1] the solution with the opposite duality was used to construct supersymmetric brane solutions in $M$-theory.

The $\tilde{v}$-system (4.11) can be solved easily and we have regular solutions but growing exponentially at infinity. Thus there are no $L^{2}$-normalizable solutions to the $\tilde{v}$-system that might give deformations to off-diagonal metrics. Though the lack of concrete examples of such metrics is a stumbling block in taking further analyses, the fact that the diagonal deformation arises from the $L^{2}$-normalizable solution suggests the corresponding global deformation does not exist.

We now turn to the second example, which is given by (4.2) with

$$
\begin{aligned}
& a^{2}=(r-m)(r+m / 2), \quad b^{2}=(r+m)(r-m / 2), \quad c_{1}^{2}=c_{2}^{2}=r^{2}, \\
& f^{2}=\frac{9 m^{2}(r+m)(r-m)}{16(r+m / 2)(r-m / 2)},
\end{aligned}
$$

and $d t=\sqrt{r^{2}-m^{2} / 4} / \sqrt{r^{2}-m^{2}} d r$. The radial coordinate $r$ runs from the singular orbit $\mathbf{C P}(2)$ at $r=m$ to infinity. The ALC metric (5.9) is obtained with the subgroup $U(1)_{1,-1}$ and therefore within the generic case $(k \neq \ell)$ of $S U(3) / U(1)_{k, \ell}$ models. Remarkably the model with this choice of $U(1)$ subgroup has recently been employed to explore conifold like geometric transitions in $\operatorname{Spin}(7)$ geometry [23]. The deformation admits only the $u$-system (4.7) with $k=-\ell=1$. Using a variable $f=b^{2} c^{2} u_{3}-a^{2} c^{2} u_{2}$ and rescaling the radial coordinate $x=r / m$, we obtain the following differential equation of Fuchsian type ;

$$
\frac{d^{4} f}{d x^{4}}+p_{1}(x) \frac{d^{3} f}{d x^{3}}+p_{2}(x) \frac{d^{2} f}{d x^{2}}+p_{3}(x) \frac{d f}{d x}+p_{4}(x) f=0
$$

where

$$
p_{1}=\frac{256 x^{8}-120 x^{6}-118 x^{4}+53 x^{2}+7}{x\left(x^{2}-1\right)\left(x^{2}-1 / 4\right)\left(32 x^{4}-12 x^{2}-7\right)},
$$




$$
\begin{aligned}
& p_{2}=\frac{64 x^{8}+296 x^{6}-246 x^{4}-61 x^{2}-14}{x^{2}\left(x^{2}-1\right)\left(x^{2}-1 / 4\right)\left(32 x^{4}-12 x^{2}-7\right)}, \\
& p_{3}=-\frac{2\left(320 x^{8}+64 x^{6}+222 x^{4}-8 x^{2}-7\right)}{x^{3}\left(x^{2}-1\right)\left(x^{2}-1 / 4\right)\left(x^{4}-12 x^{2}-7\right)}, \\
& p_{4}=\frac{156\left(8 x^{2}+1\right)}{\left(x^{2}-1\right)\left(x^{2}-1 / 4\right)\left(32 x^{4}-12 x^{2}-7\right)} .
\end{aligned}
$$

The general solution is given by

$$
\begin{aligned}
& f_{1}=\frac{x^{2}+x+1}{(x+1)\left(x^{2}-1 / 4\right)}, \quad f_{2}=\frac{x^{2}(10 x-7)}{10(x+1)\left(x^{2}-1 / 4\right)} \\
& f_{3}=\frac{(x-1)^{2}\left(4 x^{4}+12 x^{3}+31 x^{2}-9 x-3\right)}{4(x+1)\left(x^{2}-1 / 4\right)}, \quad f_{4}=\frac{1}{\left(x^{2}-1\right)\left(x^{2}-1 / 4\right)},
\end{aligned}
$$

and so the regular solution in the region $x \geq 1$ is a linear combination $f(x)=k_{1} f_{1}(x)+$ $k_{2} f_{2}(x)+k_{3} f_{3}(x)$. The $L^{2}$-normalizability of $G^{S D}$ requires the condition for the coefficients, namely $k_{2}=10 k_{1}$ and $k_{3}=0$. Finally we find

$$
\begin{aligned}
& u_{1}=-\frac{k_{1}(x-1)\left(16 x^{2}+9 x-1\right)}{3 x(x+1)^{2}\left(x^{2}+1 / 2\right)^{2}(x-1 / 2)^{2}}, \quad u_{2}=-\frac{k_{1}(x-1)\left(14 x^{2}+6 x+1\right)}{6 x^{3}(x+1)(x+1 / 2)^{2}(x-1 / 2)}, \\
& u_{3}=\frac{k_{1}\left(46 x^{4}-14 x^{3}+3 x^{2}+2 x-1\right)}{6 x^{3}(x+1)^{2}(x+1 / 2)(x-1 / 2)^{2}}, \quad u_{4}=-\frac{k_{1}(x-1)(3 x+1)}{6 x^{3}(x+1)^{2}(x+1 / 2)(x-1 / 2)} .(5.13
\end{aligned}
$$

The behavior of the metric on the $\operatorname{Spin}^{c}$ bundle near the singular orbit $\mathbf{C P}(2)$ is given by 30

$$
\begin{aligned}
& a(t)=t\left(1-\frac{1}{2}(q+1)(t / m)^{2}+\cdots\right), \\
& b(t)=m\left(1+\frac{1}{6}(4-n)(t / m)^{2}+\cdots\right), \\
& c(t)=m\left(1+\frac{1}{6}(5+n)(t / m)^{2}+\cdots\right), \\
& f(t)=t\left(1+q(t / m)^{2}+\cdots\right),
\end{aligned}
$$

where $q, m$ are free parameters and the integer $n$ represents an odd class in $H^{2}(\mathbf{C P}(2), \mathbf{Z})$ that defines a $\operatorname{Spin}^{c}$ structure of $\mathbf{C P}(2)$. The metric (5.9) corresponds to $q=-26 / 27$ with $n=-1$ and the solution (5.13) induces the transformation

$$
q=-26 / 27 \rightarrow \tilde{q}=\left(-26+40 k_{1}\right) / 27, \quad m \rightarrow \tilde{m}=m\left(1-k_{1}\right)
$$

\section{AC metric}


Here for completeness we present a summary of the results for AC metrics with special holonomy. The qualitative feature of the deformation is different, since we cannot find $L^{2}$-normalizable solutions. There are three explicitly known AC metrics based on the coset space $S U(3) / U(1)_{1,1}$ with the metric ansatz (4.2) ;

(a) $\operatorname{Sp}(2)$ metric on $T^{*} \mathbf{C P}(2)$ 25] [17]

$$
\begin{aligned}
& a^{2}=\frac{1}{2}\left(r^{2}-m^{2}\right), \quad b^{2}=\frac{1}{2}\left(r^{2}+m^{2}\right), \quad c_{1}^{2}=c_{2}^{2}=r^{2} \\
& f^{2}=\frac{r^{2}}{4}\left(1-(m / r)^{4}\right) \quad \text { with } \quad t=\int_{m}^{r} \frac{d r}{\sqrt{1-(m / r)^{4}}} .
\end{aligned}
$$

(b) $S U(4)$ metric on the line bundle over $S U(3) / T^{2}$ [26] [17]

$$
\begin{aligned}
& a^{2}=b^{2}=\frac{r^{2}}{2}, \quad c_{1}^{2}=c_{2}^{2}=r^{2} \\
& f^{2}=\frac{r^{2}}{4}\left(1-(m / r)^{8}\right) \quad \text { with } \quad t=\int_{m}^{r} \frac{d r}{\sqrt{1-(m / r)^{8}}}
\end{aligned}
$$

(c) $\operatorname{Spin}(7)$ metric on $T^{*} \mathbf{C P}(2) / \mathbf{Z}_{2}$ 17]

$$
\begin{aligned}
& a^{2}=b^{2}=\frac{9 r^{2}}{10}, \quad c_{1}^{2}=c_{2}^{2}=\frac{9 r^{2}}{25}\left(1-(m / r)^{10 / 3}\right) \\
& f^{2}=\frac{9 r^{2}}{100}\left(1-(m / r)^{10 / 3}\right) \quad \text { with } \quad t=\int_{m}^{r} \frac{d r}{\sqrt{1-(m / r)^{10 / 3}}}
\end{aligned}
$$

As before one can explicitly calculate the closed self-dual four forms $G^{S D}$ for these metrics and show that just as for the ALC metric there exists a regular $G^{S D}$ which describes deformations at least locally. However, the $L^{2}$-normalizability is lost for all examples, which suggests that the deformations are non-dynamical. From the power series expansion we see that the corresponding deformation would change the asymptotic behavior of the metric from AC type to ALC type. This analysis seems consistent with what we have found for the $L^{2}$-normalizability. We should remark that it is possible to have an $L^{2}$-normalizable solution in the anti-self-dual side. In fact this is the case for the Calabi metric on $T^{*} \mathbf{C P}(2)$ [17.

In the following we list the regular solutions to the $u$ and $v$-systems ; (a-1) $u$-system of $S p(2)$ metric 


$$
\begin{aligned}
& u_{1}=\frac{k_{1}(x-1)\left(3 x^{2}+9 x+8\right)}{(x+1)^{2}}, \\
& u_{2}=\frac{k_{1}(x-1)\left(3 x^{3}+15 x^{2}+x+1\right)}{2 x^{2}(x+1)}+\frac{5 k_{2}}{2 x^{2}}, \\
& u_{3}=\frac{k_{1}(x-1)^{3}\left(3 x^{2}+6 x+1\right)}{2 x^{2}(x+1)^{2}}-\frac{5 k_{2}(x+1)}{2 x^{2}}, \\
& u_{4}=u_{5}=-u_{1} / 2 \text { with } \quad x=(r / m)^{2} .
\end{aligned}
$$

(a-2) $v$-system of $S p(2)$ metric

$$
v_{1}=-v_{3}=\frac{k_{3}}{x}, \quad v_{2}=-v_{4}=\frac{k_{4}}{x}, \quad v_{5}=0 .
$$

(b-1) $u$-system of $S U(4)$ metric

$$
\begin{aligned}
& u_{1}=\frac{k_{1}}{x^{4}}+\frac{k_{3} w(x)}{3}, \\
& u_{2}=\frac{k_{1}}{x^{4}}+\frac{k_{2}}{x}-\frac{k_{3} w(x)}{6}, \\
& u_{3}=\frac{k_{1}}{x^{4}}-\frac{k_{2}}{x}-\frac{k_{3} w(x)}{6}, \\
& u_{4}=-u_{5}=\frac{k_{3} \sqrt{x^{4}-1}}{8} F\left[1 / 4,3 / 2,2 ; 1-x^{4}\right]
\end{aligned}
$$

and

$$
w=-x^{2} F\left[1 / 4,3 / 2,2 ; 1-x^{4}\right]+\frac{3 x^{2}\left(x^{4}-1\right)}{16} F\left[5 / 4,5 / 2,3 ; 1-x^{4}\right] .
$$

Here $x=(r / m)^{2}$ and $F[\alpha, \beta, \gamma ; x]$ denotes the hypergeometric function.

(b-2) $v$-system of $S U(4)$ metric

There are no regular solutions.

(c-1) $u$-system of $\operatorname{Spin}(7)$ metric [16]

$$
\begin{aligned}
& u_{1}=\frac{6 k_{1} z\left(z^{7 / 5}-1\right)}{z-1}, \\
& u_{2}=u_{3}=k_{1} w(z)+k_{2} z^{-4 / 5}(z-1), \\
& u_{4}=k_{1} w(z)-\frac{\left(k_{2}+k_{3}\right)}{2} z^{-4 / 5}(z-1), \\
& u_{5}=k_{1} w(z)-\frac{\left(k_{2}-k_{3}\right)}{2} z^{-4 / 5}(z-1)
\end{aligned}
$$


and

$$
w=6+z^{7 / 5}-\frac{4 z^{7 / 5}-11}{z-1}-\frac{5\left(z^{7 / 5}-1\right)}{(z-1)^{2}} \quad \text { with } \quad z=(m / r)^{10 / 3} .
$$

(c-2) $v$-system of $\operatorname{Spin}(7)$ metric

$$
v_{i}=K_{i} z^{-4 / 5}(z-1), \quad(i=1 \sim 5)
$$

where $K_{i}$ are constants satisfying $K_{1}=K_{3}$ and $K_{2}=K_{4}$.

Finally let us comment on the AC special holonomy metrics based on the coset space $S p(2) / S p(1)$. Using the correspondence between $S p(2) / S p(1)$ and $S U(3) / U(1)_{1,1}$ models, we can see the deformations of the AC metrics. In fact the solution (5.21) gives the deformation of $S U(4)$ metric on the line bundle over $\mathbf{C P}(3)$ [26] [17], and the solution (5.23) the deformation of $\operatorname{Spin}(7)$ metric on the bundle of chiral spinors over $S^{4}$ [24] [13].

\section{Acknowledgements}

We would like to thank T. Eguchi, R. Goto and M. Naka for helpful discussions. This work is supported in part by the Grant-in-Aid for Scientific Research No. 14570073.

\section{Appendix A}

\section{Convention of $S U(3)$ Maurer-Cartan forms}

We use the following $S U(3)$ Maurer-Cartan equation that is $\Sigma_{3}$ symmetric;

$$
\begin{aligned}
& d \sigma_{1}=\Sigma_{1} \wedge \tau_{1}-\Sigma_{2} \wedge \tau_{2}+\kappa_{A} T_{A} \wedge \sigma_{2}+\kappa_{B} T_{B} \wedge \sigma_{2}, \\
& d \sigma_{2}=-\Sigma_{1} \wedge \tau_{2}-\Sigma_{2} \wedge \tau_{1}-\kappa_{A} T_{A} \wedge \sigma_{1}-\kappa_{B} T_{B} \wedge \sigma_{1}, \\
& d \Sigma_{1}=\tau_{1} \wedge \sigma_{1}-\tau_{2} \wedge \sigma_{2}+\mu_{A} T_{A} \wedge \Sigma_{2}+\mu_{B} T_{B} \wedge \Sigma_{2}, \\
& d \Sigma_{2}=-\tau_{1} \wedge \sigma_{2}-\tau_{2} \wedge \sigma_{1}-\mu_{A} T_{A} \wedge \Sigma_{1}-\mu_{B} T_{B} \wedge \Sigma_{1}, \\
& d \tau_{1}=\sigma_{1} \wedge \Sigma_{1}-\sigma_{2} \wedge \Sigma_{2}+\nu_{A} T_{A} \wedge \tau_{2}+\nu_{B} T_{B} \wedge \tau_{2}, \\
& d \tau_{2}=-\sigma_{1} \wedge \Sigma_{2}-\sigma_{2} \wedge \Sigma_{1}-\nu_{A} T_{A} \wedge \tau_{1}-\nu_{B} T_{B} \wedge \tau_{1}, \\
& d T_{A}=2 \alpha_{A} \sigma_{1} \wedge \sigma_{2}+2 \beta_{A} \Sigma_{1} \wedge \Sigma_{2}+2 \gamma_{A} \tau_{1} \wedge \tau_{2}, \\
& d T_{B}=2 \alpha_{B} \sigma_{1} \wedge \sigma_{2}+2 \beta_{B} \Sigma_{1} \wedge \Sigma_{2}+2 \gamma_{B} \tau_{1} \wedge \tau_{2} .
\end{aligned}
$$


This form of the Maurer-Cartan equation is symmetric under the (cyclic) permutation of $\left(\sigma_{i}, \Sigma_{i}, \tau_{i}\right)$. From the Jacobi identity we see that the parameters $\alpha, \beta, \gamma, \kappa, \mu, \nu$, which describe the "coupling" of the Cartan generators $\left\{T_{A}, T_{B}\right\}$ satisfy

$$
\begin{gathered}
\alpha_{A}+\beta_{A}+\gamma_{A}=0, \alpha_{B}+\beta_{B}+\gamma_{B}=0, \\
\kappa_{A}=\frac{1}{\Delta}\left(\beta_{B}-\gamma_{B}\right), \kappa_{B}=-\frac{1}{\Delta}\left(\beta_{A}-\gamma_{A}\right), \mu_{A}=-\frac{1}{\Delta}\left(\alpha_{B}-\gamma_{B}\right), \\
\mu_{B}=\frac{1}{\Delta}\left(\alpha_{A}-\gamma_{A}\right), \nu_{A}=\frac{1}{\Delta}\left(\alpha_{B}-\beta_{B}\right), \nu_{B}=-\frac{1}{\Delta}\left(\alpha_{A}-\beta_{A}\right)
\end{gathered}
$$

with $\Delta=\beta_{A} \alpha_{B}-\alpha_{A} \beta_{B}$ leaving four free parameters $\left(\alpha_{A, B}, \beta_{A, B}\right)$. We may further put the "orthogonality" conditions;

$$
\begin{aligned}
& \alpha_{A} \alpha_{B}+\beta_{A} \beta_{B}+\gamma_{A} \gamma_{B}=0, \\
& \kappa_{A} \kappa_{B}+\mu_{A} \mu_{B}+\nu_{A} \nu_{B}=0,
\end{aligned}
$$

which reduces one parameter.

\section{Appendix B}

\section{Hitchin formulation of $\operatorname{Spin}(7)$ manifolds}

Recently Hitchin has shown that any hypersurface in a foliation of eight-manifold with $\operatorname{Spin}(7)$ holonomy carries a cosymplectic $G_{2}$-structure [18]. Here we describe the outline restricting to $\operatorname{Spin}(7)$ manifolds of cohomogeneity one.

Let $M=G / K$ be a seven dimensional homogeneous space with $G$-invariant metric $\hat{g}$. Explicitly, using a basis of invariant 1-forms $E^{i}$, we write

$$
\hat{g}=g_{i j} E^{i} \otimes E^{j} .
$$

A $G_{2}$-structure on $M$ is specified by fixing a three-form $\varphi$ which takes the form

$$
\varphi=\frac{1}{3 !} \varphi_{i j k} e^{i} \wedge e^{j} \wedge e^{k} .
$$

Here, $e^{i}(i=1 \sim 7)$ denote the vielbeins of $\hat{g}$, which are identified with the generators of octonions obeying the relation

$$
e^{i} e^{j}=\varphi_{i j k} e^{k}-\delta_{i j} .
$$


Furthermore $(\mathbb{B . 2})$ defines a cosymplectic $G_{2}$-structure on $M$ in case $d * \varphi=0$. Both weak $G_{2}$ and $G_{2}$ holonomy structures satisfy this condition and so are special examples of cosymplectic $G_{2}$-structures. A classification of compact homogeneous manifolds with weak $G_{2}$ holonomy is given in 21] and the spaces $S p(2) / S p(1)$ and $S U(3) / U(1)_{k \ell}$ we discuss in this paper are in the members of this list.

Suppose $\rho_{t}=* \varphi(t)$ is a closed four-form in the space of $G$-invariant cosymplectic $G_{2}$-structures on $M$ for each $t \in I$ (open interval). If

$$
\dot{\rho}_{t}+d * \rho_{t}=0
$$

then the four-form

$$
\Omega=d t \wedge * \rho_{t}-\rho_{t}
$$

gives a cohomogeneity-one metric with $\operatorname{Spin}(7)$ holonomy on the eight-manifols $N=$ $I \times M$. In fact, we have

$$
d \Omega=-d t \wedge d * \rho_{t}-d t \wedge \dot{\rho}_{t}=0
$$

The invariant metric $\hat{g}(t)$ on $M$ evolves via the equation (B.4) and induces the following $\operatorname{Spin}(7)$ metric

$$
\begin{aligned}
g & =d t^{2}+\hat{g}(t) \\
& =d t^{2}+g_{i j}(t) E^{i} \otimes E^{j} .
\end{aligned}
$$

Note that $(\mathbb{B . 4})$ can be interpreted as the gradient flow equation of the total volume

$$
\sqrt{\operatorname{det} g_{i j}(t)} \int E^{1} \wedge E^{2} \wedge \cdots \wedge E^{7}
$$

when it is regarded as the functional of the closed form $\rho_{t}$.

Conversely, if a $\operatorname{Spin}(7)$ manifold is foliated by homogeneous space $G / K$, we can write the $\operatorname{Spin}(7)$ 4-form in the form $d t \wedge * \rho_{t}-\rho_{t}$ with (B.4) and $G / K$ carries an invariant cosymplectic $G_{2}$-structure $\rho_{t}$ for each $t$.

\section{Appendix $\mathrm{C}$}

\section{Deformation of four dimensional hyperkähler manifolds}


A Riemannian manifold $(M, g)$ is called hyperkähler when it satisfies the following conditions: (a) $M$ admits three complex structures $J^{a}(a=1,2,3)$ obeying the quaternionic relations $J^{a} J^{b}=-\delta_{a b}-\epsilon_{a b c} J^{c}$; (b) the metric $g$ is preserved by $J^{a}$; (c) the two forms $\Omega^{a}$ defined by $\Omega^{a}(X, Y)=g\left(J^{a} X, Y\right)$ for all vector fields $X, Y$ are three Kähler forms; $d \Omega^{a}=0$. If the manifold is four dimensional, the above condition is equivalent to the (anti-)self-duality of the curvature two form.

In this appendix we discuss deformations of four dimensional hyperkähler manifold by a similar approach to $\operatorname{Spin}(7)$ manifold in this paper. Let us first recall a formulation of the self-dual equations by Ashtekar. In this approach hyperkähler metrics are given by solutions to the differential equations for volume-preserving vector fields. The following proposition summarizes the result of [27] 28] [29] relevant to our calculation.

Proposition Let $(M, \omega)$ be a four dimensional manifold with a volume form $\omega$ and let $\left(V_{0}, V_{1}, V_{2}, V_{3}\right)$ be volume-preserving vector fields forming an oriented basis for $T M$ at each point. Suppose in addition that the vector fields satisfy the equations

$$
\left[V_{0}, V_{1}\right]+\left[V_{2}, V_{3}\right]=0, \quad\left[V_{0}, V_{2}\right]+\left[V_{3}, V_{1}\right]=0, \quad\left[V_{0}, V_{3}\right]+\left[V_{1}, V_{2}\right]=0 .
$$

Then the following metric $g$ is hyperkähler

$$
g=e^{\mu} \otimes e^{\mu}, \quad e^{\mu}=\sqrt{\phi} V^{\mu},
$$

where $V^{\mu}$ is the dual one form of $V_{\mu}$ and $\phi$ is a function defined by $\omega=\phi V^{0} \wedge V^{1} \wedge V^{2} \wedge V^{3}$, and the hyperkähler forms are given by the self-dual two forms ;

$$
\Omega_{S D}^{1}=e^{0} \wedge e^{1}+e^{2} \wedge e^{3}, \quad \Omega_{S D}^{2}=e^{0} \wedge e^{2}+e^{3} \wedge e^{1}, \quad \Omega_{S D}^{3}=e^{0} \wedge e^{3}+e^{1} \wedge e^{2} .
$$

We note that the duality of $\Omega_{S D}^{i}$ is fixed by the orientation of the basis $V_{\mu}$ and the condition (C.1).

Remarks 1. By using the orthonormal basis $e^{\mu}$ the spin connection $\omega_{\mu \nu}$ satisfies the equations

$$
\omega_{01}+\omega_{23}=0, \quad \omega_{02}+\omega_{31}=0, \quad \omega_{03}+\omega_{12}=0,
$$

and hence the corresponding curvature two form is automatically anti-self-dual. The curvature two form has the reversed duality to the hyperkähler two forms. 
2. The volume form of the metric is $\omega_{g}=\phi^{2} V^{0} \wedge V^{1} \wedge V^{2} \wedge V^{3}$, which is different from the original volume form $\omega$ one might expect.

Conversely, it is known that the volume-preserving vector fields satisfying (C.1) can be locally constructed for any four dimensional hyperkähler manifold [29].

We now proceed to deformations of the hyperkähler manifold $(M, g)$, and use the orthonormal basis $e^{\mu}$ defined in the proposition. Let $F_{A S D}$ be an anti-self-dual closed two form (an ASD $U(1)$ instanton) on $M$;

$$
F_{A S D}=F_{1}\left(e^{0} \wedge e^{1}-e^{2} \wedge e^{3}\right)+F_{2}\left(e^{0} \wedge e^{2}-e^{3} \wedge e^{1}\right)+F_{3}\left(e^{0} \wedge e^{3}-e^{1} \wedge e^{2}\right)
$$

Let $\Omega_{S D}^{a}(a=1,2,3)$ denote the hyperkähler forms given by (C.3). We define deformed two forms by adding $F_{A S D}$ with a small parameter $\epsilon$;

$$
\tilde{\Omega}^{a}=\Omega_{S D}^{a}+\epsilon F_{A S D}
$$

Note that the shift is common to all the directions $(a=1,2,3)$. When we introduce a new basis

$$
\tilde{e}^{\mu}=e^{\mu}+\frac{\epsilon}{2} U^{\mu \nu} e^{\nu}, \quad\left(U^{\mu \nu}=U^{\nu \mu}\right)
$$

defined by

$$
\begin{aligned}
& U^{01}=F_{2}-F_{3}, \quad U^{02}=-F_{1}+F_{3}, \quad U^{03}=F_{1}-F_{2}, \\
& U^{11}=F_{1}-F_{2}-F_{3}, \quad U^{22}=-F_{1}+F_{2}-F_{3}, \quad U^{33}=-F_{1}-F_{2}+F_{3}, \\
& U^{12}=F_{1}+F_{2}, \quad U^{13}=F_{1}+F_{3}, \quad U^{23}=F_{2}+F_{3}
\end{aligned}
$$

with

$$
U^{00}=-\sum_{i=1}^{3} U^{i i}=F_{1}+F_{2}+F_{3},
$$

it is easy to confirm that $\tilde{\Omega}^{a}$ takes the same form as $\Omega_{S D}^{a}$ by means of the new basis $\tilde{e}^{\mu}$. Thus the deformed metric $\tilde{g}=\tilde{e}^{\mu} \otimes \tilde{e}^{\mu}$ is hyperkähler. Note that $F_{A S D}$ can be constructed from a volume-preserving vector field $W=W_{\mu} V_{\mu}$ satisfying the equation

$$
\left[V_{\mu},\left[V_{\mu}, W\right]\right]=0 .
$$


In fact the components of $F_{A S D}$ are given by [30]

$$
\begin{aligned}
& F_{1}=V_{1}\left(W_{0}\right)-V_{0}\left(W_{1}\right)+V_{2}\left(W_{3}\right)-V_{3}\left(W_{2}\right), \\
& F_{2}=V_{2}\left(W_{0}\right)-V_{0}\left(W_{2}\right)+V_{3}\left(W_{1}\right)-V_{1}\left(W_{3}\right), \\
& F_{3}=V_{3}\left(W_{0}\right)-V_{0}\left(W_{3}\right)+V_{1}\left(W_{2}\right)-V_{2}\left(W_{1}\right) .
\end{aligned}
$$

As an example let us consider the Atiyah-Hitchin metric [31. In [32 [33] it was shown that there exists a one-parameter family of deformations of the metric by taking a hyperkähler quotient of a moduli space of $S U(3)$ monopoles. By applying our method to this problem, the deformed metric can be made more explicit, although the expression is restricted to a small deformation from the Atiyah-Hitchin metric.

In the proposition, we take $M=\mathbf{R} \times S O(3)$ and introduce the left-invariant one forms $\sigma^{i}(i=1,2,3)$ on $S O(3)$ given by $A^{-1} d A=\sigma^{i} E_{i}, A \in S O(3)$. Here, $E_{i}$ is the basis of $s o(3)$ algebra,

$$
E_{1}=\left(\begin{array}{ccc}
0 & 0 & 0 \\
0 & 0 & 1 \\
0 & -1 & 0
\end{array}\right), E_{2}=\left(\begin{array}{ccc}
0 & 0 & -1 \\
0 & 0 & 0 \\
1 & 0 & 0
\end{array}\right), E_{3}=\left(\begin{array}{ccc}
0 & 1 & 0 \\
-1 & 0 & 0 \\
0 & 0 & 0
\end{array}\right) .
$$

Using Euler angles $\alpha, \beta$ and $\gamma$, we write

$$
A=e^{\alpha E_{1}} e^{\beta E_{2}} e^{\gamma E_{1}}
$$

and then

$$
\begin{aligned}
& \sigma^{1}=d \gamma+\cos \beta d \alpha, \\
& \sigma^{2}=\sin \beta \cos \gamma d \alpha-\sin \gamma d \beta, \\
& \sigma^{3}=\sin \beta \sin \gamma d \alpha+\cos \gamma d \beta .
\end{aligned}
$$

Let us consider vector fields on $M$ [34]

$$
V_{0}=\frac{\partial}{\partial t}, \quad V_{i}=A_{i j} \omega_{j}(t) \sigma_{j},
$$

where $A_{i j}$ represent the components of the matrix (C.13) and $\sigma_{j}$ is the dual vector field of $\sigma^{j}$. The functions $\omega_{j}(t)$ are to be determined. The vector fields $V_{\mu}$ preserve a volume 
form $\omega=d t \wedge \sigma^{1} \wedge \sigma^{2} \wedge \sigma^{3}$. So applying the proposition to $V_{\mu}$, one can obtain an $\mathrm{SO}(3)$-invariant hyperkähler metric of Bianchi IX type ;

$$
g=\omega_{1} \omega_{2} \omega_{3} d t^{2}+\frac{\omega_{2} \omega_{3}}{\omega_{1}} \sigma^{1} \otimes \sigma^{1}+\frac{\omega_{1} \omega_{3}}{\omega_{2}} \sigma^{2} \otimes \sigma^{2}+\frac{\omega_{1} \omega_{2}}{\omega_{3}} \sigma^{3} \otimes \sigma^{3} .
$$

and the condition (C.1) implies that $\omega_{i}$ must satisfy the equation

$$
\dot{\omega}_{1}=\omega_{2} \omega_{3}-\omega_{1}\left(\omega_{2}+\omega_{3}\right)
$$

and its cyclic permutations. The flow equation (C.17) can be solved in terms of elliptic functions, which gives the Atiyah-Hitchin metric. An $S O(3)$-invariant closed anti-selfdual two form $F_{A S D}$ on the Atiyah-Hitchin manifold (sometimes called the Sen form) was analysed by [35] [36], and it takes the form

$$
F_{A S D}=f(t)\left(\omega_{2} \omega_{3} d t \wedge \sigma^{1}-\omega_{1} \sigma^{2} \wedge \sigma^{3}\right)
$$

where

$$
f(t)=\exp \int_{0}^{t}\left(\omega_{2}+\omega_{3}-2 \omega_{2} \omega_{3} / \omega_{1}\right) d t
$$

The function $f$ has an exponential decline at $t \rightarrow \infty$ showing the $L^{2}$-normalizability of $F_{A S D}$. If we rewrite (C.18) using the orthonormal basis $e^{\mu}=\sqrt{\omega_{1} \omega_{2} \omega_{3}} V^{\mu}$ defined by the proposition, then the components $F_{a}(a=1,2,3)$ of C.5 depend on Euler angles through the functions $A_{i j}$. In fact

$$
F_{1}=f(t) A_{11}, \quad F_{2}=f(t) A_{21}, \quad F_{3}=f(t) A_{31}
$$

This breaks the $S O(3)$ symmetry of the Atiyah-Hitchin metric when the metric is deformed by $F_{A S D}$. Combining (C.20) with (C.7), we have an expression for the deformed metric $\tilde{g}=g+\epsilon h$;

$$
\begin{aligned}
h & =\left(A_{11}+A_{21}+A_{31}\right) f\left(\omega_{1} \omega_{2} \omega_{3} d t^{2}+\frac{\omega_{2} \omega_{3}}{\omega_{1}} \sigma^{1} \otimes \sigma^{1}-\frac{\omega_{1} \omega_{3}}{\omega_{2}} \sigma^{2} \otimes \sigma^{2}-\frac{\omega_{1} \omega_{2}}{\omega_{3}} \sigma^{3} \otimes \sigma^{3}\right) \\
& +\left(A_{12}+A_{22}+A_{32}\right) f\left(\omega_{1} \omega_{2}\left(d t \otimes \sigma^{3}+\sigma^{3} \otimes d t\right)+\omega_{3}\left(\sigma^{1} \otimes \sigma^{2}+\sigma^{2} \otimes \sigma^{1}\right)\right) \\
& +\left(A_{13}+A_{23}+A_{33}\right) f\left(-\omega_{1} \omega_{3}\left(d t \otimes \sigma^{2}+\sigma^{2} \otimes d t\right)+\omega_{2}\left(\sigma^{1} \otimes \sigma^{3}+\sigma^{3} \otimes \sigma^{1}\right)\right) . \quad(\text { C. } 21)
\end{aligned}
$$

Let $K_{i}(i=1,2,3)$ be vector fields on $S O(3)$ generated by the left transformation $\exp \left(t E_{i}\right)$. Then the functions $A_{1 j}+A_{2 j}+A_{3 j}$ vanish under the action $K=K_{1}+K_{2}+K_{3}$. Thus the metric $\tilde{g}$ has an isometry $S O(2)$ generated by $K$ as has been claimed in [33]. 


\section{References}

[1] M. Cvetič, G.W. Gibbons, H. Lü and C.N. Pope, New Complete Non-compact Spin(7) Manifolds, Nucl. Phys. B620 (2002) 29, hep-th/0103155; New Cohomogeneity One Metrics with Spin(7) Holonomy, math.DG/0105119.

[2] A. Brandhuber, J. Gomis, S.S. Gubser and S. Gukov, Gauge Theory at Large N and New $G_{2}$ Holonomy Metrics, Nucl. Phys. B611 (2001) 179, hep-th/0106034.

[3] M. Cvetič, G.W. Gibbons, H. Lü and C.N. Pope, Cohomogeneity One Manifolds of $\operatorname{Spin}(7)$ and $G_{2}$ Holonomy, Phys. Rev. D65 (2002) 106004, hep-th/0108245.

[4] S. Gukov and J. Sparks, $M$-Theory on Spin(7) Manifolds: I, Nucl. Phys. B625 (2002) 3, hep-th/0109025.

[5] H. Kanno and Y. Yasui, On Spin(7) Holonomy Metric Based on $S U(3) / U(1)$, hep-th/0108226 (to appear in J. Geom. Phys).

[6] H. Kanno and Y. Yasui, On Spin(7) Holonomy Metric Based on $S U(3) / U(1)$ II, hep-th/0111198 (to appear in J. Geom. Phys).

[7] M. Cvetič, G.W. Gibbons, H. Lü and C.N. Pope, M-theory Conifolds, Phys. Rev. Lett. 88 (2002) 121602, hep-th/0112098; A $G_{2}$ Unification of the Deformed and Resolved Conifolds, Phys. Lett. B534 (2002) 172, hep-th/0112138.

[8] B.S. Acharya, On realising $N=1$ super Yang-Mills in $M$ theory, hep-th/0011089.

[9] M. Atiyah, J. Maldacene and C. Vafa, An $M$-theory flop as a large $N$ Duality, J. Math. Phys. 42 (2001) 3209, hep-th/0011256.

[10] M. Atiyah and E. Witten, $M$-theory dynamics on a manifold of $G_{2}$ holonomy, hep-th/0107177.

[11] B.S. Acharya, $M$ theory, Joyce orbifolds and super Yang-Mills, Adv. Theor. Math. Phys. 3 (1999) 227, hep-th/9812205.

[12] D.D. Joyce, Compact Manifolds with Special Holonomy, Oxford University Press (2000). 
[13] G.W. Gibbons, D.N. Page and C.N. Pope, Einstein metrics on $S^{3}, R^{3}$ and $R^{4}$ Bundles, Commun. Math. Phys. 127 (1990) 529.

[14] T. Hausel, E. Hunsicker and R. Mazzeo, Hodge cohomology of gravitational instantons, math.DG/0207169.

[15] B.S. Acharya, X. de la Ossa and S. Gukov, G-Flux, Supersymmetry and Spin(7) Manifolds, hep-th/0201227.

[16] M. Cvetič, H. Lü and C.N. Pope, Brane Resolution Through Transgression, Nucl. Phys. B600 (2001) 103, hep-th/0011023.

[17] M. Cvetič, G.W. Gibbons, H. Lü and C.N. Pope, Hyper-Kähler Calabi Metrics, $L^{2}$ Harmonic Forms, Resolved M2-branes and $A d S_{4} / C F T_{3}$ Correspondence, hep-th/0102185.

[18] N. Hitchin, Stable Forms and Special Metrics, math.DG/0107101.

[19] S. Gukov, S.-T. Yau and E. Zaslow, Duality and Fibrations on $G_{2}$ manifolds, hep-th/0203217.

[20] A. Brandhuber, $G_{2}$ Holonomy Space from Invariant Three-Forms, hep-th/0112113.

[21] T. Friedrich, I. Kath, A. Moroianu and U. Semmelmann, On nealy parallel $G_{2^{-}}$ structures, J. Geom. Phys. 23 (1997)259.

[22] K. Becker, A note on compactifications on Spin(7)-holonomy manifolds, JHEP 0011 (2000) 029, hep-th/0011114.

[23] S. Gukov, J. Sparks and D. Tong, Conifold Transitions and Five-Brane Condensation in $M$-Theory on $\operatorname{Spin}(7)$ Manifolds, hep-th/0207244.

[24] R. Bryant and S. Salamon, On the construction of some complete metrics with exceptional holonomy, Duke Math. J. 58 (1989) 829.

[25] A. Dancer and A. Swann, Hyperkähler metrics of cohomogeneity one, J. Geom. Phys. 21 (1997) 218. 
[26] D.N. Page and C.N. Pope, Inhomogeneous Einstein Metrics on Complex Line Bundles, Class. Quantum Grav. 4 (1987) 213.

[27] A. Ashtekar, T. Jacobson and L. Smolin, A new characterization of half-flat solutions to Einstein's equation, Commun. Math. Phys. 115 (1988) 631.

[28] L.J. Mason and E.T. Newman, A connection between the Einstein and Yang-Mills equations, Commun. Math. Phys. 121 (1989) 659.

[29] S.K. Donaldson, Complex cobordism, Ashtekar's equations and diffeomorphisms, Symplectic Geometry, edited by D. Salamon, London Math. Soc., London, (1992) 44.

[30] T. Ootsuka, S. Miyagi, Y. Yasui and S. Zeze, Anti-self-dual Maxwell solutions on hyperkähler manifold and $\mathrm{N}=2$ supersymmetric Ashtekar gravity, Class. Quantum Grav. 16 (1999) 1305.

[31] M.F. Atiyah and N.J. Hitchin, The geometry and dynamics of magnetic monopoles, Princeton University Press, Princeton (1988).

[32] A.S. Dancer, Nahm's equations and hyperkähler geometry, Commnu. Math. Phys. 158 (1993) 545.

[33] A.S. Dancer, A family of hyperkähler manifolds, Quart. J. Math. 45 (1994) 463.

[34] M.J. Ablowitz and P.A. Clarkson, Solitons, nonlinear evolution equations and inverse scattering, London Math. Soc. Lecture Notes in Mathematics, 149 (1991), Cambridge University Press, Cambridge.

[35] G.W. Gibbons and P.J.Ruback, The hidden symmetries of multi-centre metrics, Commnu. Math. Phys. 115 (1988) 267.

[36] A. Sen, Dyon-monopole bound states, self-dual harmonic forms on the multimonopole moduli space, and SL(2,Z) invariance in string theory, Phys. Lett. 329B (1994) 217. 